\title{
Could changes in national tuberculosis vaccination policies be ill-informed?
}

\author{
D. J. Gerberry ${ }^{1 *}$, F. A. Milner ${ }^{2}$ \\ ${ }^{1}$ Department of Mathematics and Computer Science, Xavier University, \\ 3800 Victory Parkway, Cincinnati, Ohio 45207, USA \\ ${ }^{2}$ School of Mathematical and Statistical Sciences, Arizona State University, \\ P.O. Box 871804, Tempe, Arizona 85287, USA
}

\begin{abstract}
National policies regarding the BCG vaccine for tuberculosis vary greatly throughout the international community and several countries are currently considering discontinuing universal vaccination. Detractors of BCG point to its uncertain effectiveness and its interference with the detection and treatment of latent tuberculosis infection (LTBI).

In order to quantify the trade-off between vaccination and treatment of LTBI, a mathematical model was designed and calibrated to data from Brazil, Ghana, Germany, India, Mexico, Romania, the United Kingdom and the United States. Country-specific thresholds for when LTBI treatment outperforms mass vaccination were found and the consequences of policy changes were estimated.

Our results suggest that vaccination outperforms LTBI treatment in all settings but with greatly reduced efficiency in low incidence countries. While national policy statements emphasize BCG's interference with LTBI detection, we find that reinfection should be more determinant of a country's proper policy choice.
\end{abstract}

Keywords and phrases: tuberculosis, BCG, vaccination, mathematical modeling, threshold analysis, latent tuberculosis detection, latent tuberculosis treatment

Mathematics Subject Classification: 92B05, 37N25

\section{Introduction}

The July 2007 CDC issue of TB Elimination [1] begins by stating that "Treatment of latent TB infection (LTBI) is essential to controlling and eliminating TB in the United States." At the same time, the World Health Organization (WHO) recommends vaccination with Bacille Calmette-Guérin (BCG) of all healthy infants in countries with high TB burdens [2]. Hence, both BCG vaccination and treatment of LTBI have an important role to play in the global fight against tuberculosis. Unfortunately, a tradeoff exists between BCG vaccination and treatment of LTBI as "the reactivity to tuberculin that occurs after vaccination interferes with the management of persons who are possibly infected with M. tuberculosis," CDC [3]. Given the lack of definitive estimates for vaccine efficacy and latent TB detection rates, mathematical

*Corresponding author. E-mail: david.gerberry@xavier.edu 

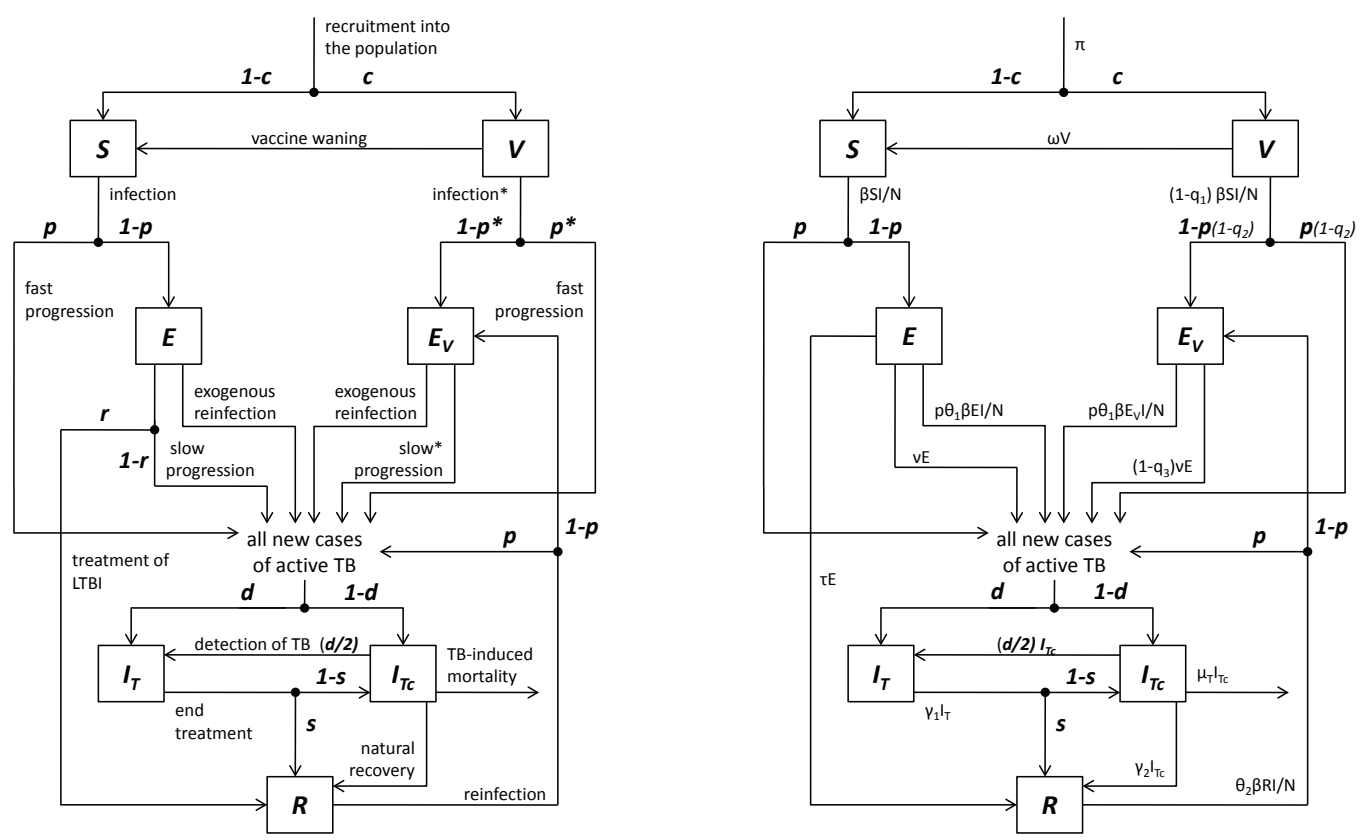

Figure 1. Flow diagram of the model. All parameters of the model are positive constants. The total number of infectious individuals is given by $I=I_{T}+I_{T^{c}}$ and $N$ is the total population size. Natural mortality (which affects individuals in all classes) and immigration are omitted from diagram for clarity of presentation.

modeling provides an appropriate and valuable perspective from which to examine this tradeoff as it includes the flexibility necessary for testing many scenarios.

In this work, we use mathematical modeling to answer three important questions regarding the tradeoff between vaccination and detection/treatment of LTBI:

1). When does vaccination increase/decrease TB incidence?,

2). How much better would one vaccination policy be?,

3). When is mass vaccination justified?

Moreover, this data-based model provides a framework for making informed policy decisions based on quantitative estimates of expected incidence and prevalence for a given population.

\section{Mathematical model}

One of the most important qualities of our mathematical model is its data-based nature. By data-based, we mean that it was formulated after considering the available data for TB so that disease indicators could be directly imported into the system. Doing so allows the model to be calibrated to data from eight countries with varying disease burdens: three low-burden countries (Germany, the United Kingdom and the United States) and five higher-burden countries (Brazil, Ghana, India, Mexico and Romania). Country-specific vaccine coverage as well as active TB detection and treatment rates were obtained from WHO data $[4,5]$. Demographic and migration parameters were derived using data from [6].

As depicted in Figure 1, the mathematical model separates the population into seven disjoint classes based on disease status: susceptible $(S)$, exposed, i.e. latently infected $(E)$, vaccinated $(V)$, vaccinated and latently infected $\left(E_{V}\right)$, infectious (i.e. active TB) and treated $\left(I_{T}\right)$, infectious and untreated $\left(I_{T^{c}}\right)$, and recovered $(R)$.

Individuals enter the population with a birth rate of $\pi$, of which a proportion, $c$, are vaccinated as newborns. As is well-established for BCG, the protective effect of the vaccine wanes over time at a rate 
which we denote $\omega$. We use the frequency-dependent description of disease transmission, with transmission coefficient $\beta$. Using the terminology of [7], we have that upon initial infection with $M$. tuberculosis, a proportion, $p$, of individuals will exhibit "fast progression" to active TB, while the remainder develop latent infection. Latent infections can progress to active disease via either reactivation of the latent infection which we term "slow progression" or a novel external infection known as exogenous reinfection [8, 9]. We denote the rate of slow progression from latent to active TB infection as $\nu$ and model exogenous reinfection via frequency-dependent transmission. We assume that previous latent infection provides protection against exogenous reinfection [9] and thus multiply the transmission term by a factor $\theta_{1} \leq 1$. Again, only a proportion, $p$, of such cases will undergo fast progression.

The model allows for vaccination to confer protection via three methods: by preventing initial infection $\left(q_{1}\right)$, by reducing the probability of fast progression to active TB $\left(q_{2}\right)$, and by reducing the rate of slow progression from latent to active TB $\left(q_{3}\right)$. We refer to $q_{1}, q_{2}$, and $q_{3}$ as efficacies of the vaccine and have that $0 \leq q_{1}, q_{2}, q_{3} \leq 1$, where $q_{i}=1$ denotes perfect protection. To incorporate these protective effects, we multiply the rate of transmission, the probability of fast progression, and the rate of slow progression by $1-q_{1}, 1-q_{2}$, and $1-q_{3}$, respectively. When thinking of vaccination, the protection described by $q_{1}$ is usually the first which comes to mind. However, there is evidence that BCG protects against progression to active TB $\left(q_{2}, q_{3}\right)$ as well [10] and that this may even be the principal mode of protection [11]. It is believed that BCG confers no additional protection against exogenous reinfection beyond preventing initial infection [10].

To formalize the trade off between vaccination and detection of latent TB, we make the pessimistic assumption that latent infection in vaccinated individuals is completely undetectable and hence untreatable. This assumption is almost surely overly pessimistic as evidence suggests that tuberculin reactivity wanes in individuals vaccinated as infants $[12,13]$ and that tuberculin skin testing can still be a useful tool for BCG-vaccinated individuals [14-19]. While still quite expensive and not widely used, diagnostic tools known as interferon-gamma release assays (IGRAs) that are not confounded by previous BCG vaccination are becoming available [20-22]. The reason for taking such a pessimistic stance on BCG is grounded in the current trend of countries moving away from universal vaccination. Hence, recommendations against the prevailing trend should only be made if they stand up to the strictest assumptions against BCG.

We assume that latently infected individuals who are not vaccinated are detected and successfully treated at a rate $\tau$ and hence move to the recovered class. The value of $\tau$ is prescribed so that a proportion, $r$, of latent infections are detected and treated successfully before progression to active disease or death. This reduces to having $\tau=\frac{r}{1-r}(\mu+\nu)$. The proportion, $r$, is favored as a model parameter as opposed to rate, $\tau$, because of its intuitive definition and consistency with the corresponding treatment indicators for active disease. As reliable estimates do not exist for detection and treatment of LTBI, we aggregate them into one indicator $r$.

For active disease, we separate the aspects of detection and treatment success into separate parameters $d$ and $s$, respectively, in order to preserve consistency with disease indicators reported in the World Health Organization's Global TB Database [4]. We assume that a proportion, $d$, of all new cases of active TB are detected and hence treated. To achieve reasonable durations of active infection, existing cases of active disease are detected at a rate of $d / 2$. Individuals complete treatment at a rate of $\gamma_{1}$ where a proportion, $s$, have successfully cured the patient. The remaining individuals move to the untreated class. We assume a disease-induced death rate of $\mu_{T}$ that only affects those with untreated active TB and that, left untreated, the disease clears naturally at a rate of $\gamma_{2}$. Lastly, we assume that reinfection is possible for individuals who have recovered from active TB. While it is biologically plausible that partial protection would be conferred, there is evidence suggesting that previous active disease may actually increase susceptibility to future TB infection [23]. Hence, we allow for reinfection of recovered individuals which is scaled by a factor $\theta_{2}\left(\theta_{2}<1\right.$ represents protection and $\theta_{2}>1$ increased susceptibility).

In many countries of Western Europe and North America, immigration plays a key role in the dynamics of tuberculosis. In the United States for example, foreign-born individuals accounted for $58 \%$ of all new TB cases reported in 2007 [24]. While omitted in Figure 1 for clarity, immigration is included in the 
mathematical model by allowing for the flow of individuals into the $S, E, V$ and $E_{V}$ classes (i.e. we assume that actively infected and recovered individuals are prevented from immigrating). For further description of the model see [25].

With the rates of movement between classes established, one can easily write down the system of differential equations that formalizes our mathematical model:

$$
\begin{aligned}
S^{\prime}= & (1-\alpha)(1-c) \pi+\alpha(1-\phi)(1-\psi) \pi+\omega V-\beta S I / N-\mu S, \\
V^{\prime}= & c(1-\alpha) \pi+\alpha \pi(1-\psi) \phi-\omega V-\left(1-q_{1}\right) \beta V I / N-\mu V, \\
E^{\prime}= & (1-p) \beta S I / N+\alpha \pi(1-\phi) \psi-\left(\nu E+\theta_{1} p \beta E I / N\right)-\tau E-\mu E, \\
E_{v}{ }^{\prime}= & {\left[1-p\left(1-q_{2}\right)\right]\left(1-q_{1}\right) \beta V I / N+\alpha \pi \psi \phi+(1-p) \theta_{2} \beta R I / N } \\
& -\left[\left(1-q_{3}\right) \nu E_{v}+\theta_{1} p \beta E_{v} I / N\right]-\mu E_{v}, \\
I_{T}{ }^{\prime}= & d\left[\left(\nu E+\theta_{1} p \beta E I / N\right)+p \beta S I / N+p\left(1-q_{2}\right)\left(1-q_{1}\right) \beta V I / N\right. \\
& \left.+\left(1-q_{3}\right) \nu E_{v}+\theta_{1} p \beta E_{v} I / N+p \theta_{2} \beta R I / N\right]-\gamma_{1} I_{T}-\mu I_{T}+\frac{d}{2} I_{T^{c}}, \\
I_{T^{c}}{ }^{\prime}= & (1-d)\left[\left(\nu E+\theta_{1} p \beta E I / N\right)+p \beta S I / N+p\left(1-q_{2}\right)\left(1-q_{1}\right) \beta V I / N+\left(1-q_{3}\right) \nu E_{v}\right. \\
& \left.+\theta_{1} p \beta E_{v} I / N+p \theta_{2} \beta R I / N\right]+(1-s) \gamma_{1} I_{T}-\gamma_{2} I_{T^{c}}-\left(\mu+\mu_{T}\right) I_{T^{c}}-d / 2 I_{T^{c}}, \\
R^{\prime}= & s \gamma_{1} I_{T}+\gamma_{2} I_{T^{c}}+\tau E-\theta_{2} \beta R I / N-\mu R,
\end{aligned}
$$

where $I=I_{T}+I_{T^{c}}$ is the total number of actively infected individuals, $N$ is the size of the total population and $\tau=\frac{r}{1-r}(\nu+\mu)$.

\section{Calibration to real data}

For each of the eight countries, the model is able to simultaneously achieve a good fit to the WHOreported rates for both incidence and prevalence of active TB (see Figure 2). Mathematically, this is not surprising as the numerous model parameters provide many degrees of freedom with which to match a limited number of data points. However, the method used to calibrate the model to data was specifically designed to buffer this fact.

To begin, estimates for several model parameters were derived from the literature and universally fixed throughout the study (See Table 1). A few elementary observations are helpful along these lines. If individuals leave a compartment with some rate $x$, then the amount of time an average individual spends in the compartment is exponentially distributed with a mean of $1 / x$. For example, the natural mortality rate $\mu$ can be easily approximated because $1 / \mu$ is the average life expectancy. The connection to exponential distributions also allows us to approximate $\nu$. In this case, latent infections progress slowly to active TB at a rate $\nu$ whereas natural mortality occurs at a rate $\mu$. Given that the probability of progressing to active disease before death is given by $\frac{\nu}{\nu+\mu}$, estimates of this probability and the natural mortality rate provide a quick estimate for $\nu$. The same relationship is used to relate the treatment rate of LTBI $(\tau)$ to the proportion of latently infected individuals who are detected and successfully treated before progression to active disease or death. Without exogenous reinfection, the probability is given by $\frac{\tau}{\tau+\mu+\nu}$. Setting this equal to $r$, we find that the appropriate treatment rate is $\tau=\frac{r}{1-r}(\nu+\mu)$.

Demographic parameters were derived using data from [6]. Estimates for average life expectancy give immediate values for the mortality parameter $\mu$. To obtain an estimate for the recruitment parameter $\pi$, we use the equation for the total population size, $N$. Note that, in the absence of TB-induced mortality, $N^{\prime}=\pi-\mu N$, which has solution $N(t)=\pi / \mu+\left(N_{0}-\pi / \mu\right) e^{-\mu t}$, where $N_{0}$ denotes the initial population size. Using estimated values for $\mu$ and $N_{0}$, we find the value of $\pi$ that results in the statistical best fit to the reported data. For immigration parameter $\alpha$, we use data for total births and net migration from [6] in order to find the percentage of total recruitment due to immigration. See Table 2 for the resulting estimates. With several model parameters now fixed, there are significantly fewer degrees of freedom to calibrate the model to data. 
TABLE 1. Parameter values and distributions used for all countries in the study. Parameters with one value are fixed and used in all simulations. Parameters with only a minimum and maximum value are sampled using uniform distributions. Parameters with a minimum, mode and maximum value are sampled using triangular distributions. ${ }^{a}$ Estimates of the probability of progression to active infection within a few years (i.e. primary disease) range from 5-10\% [9]. We assume $5 \%$ as our system models fast progression immediately after initial infection. ${ }^{b}[20,26-$ 28] estimate a $5-10 \%$ lifetime risk of an infected individual developing active TB. $\omega$ is chosen so that the probability of reactivation before death (i.e. $\frac{\nu}{\nu+\mu}$ ) is 0.05 , assuming an average life expectancy of 70 years. Given a $5 \%$ chance of fast progression and a $5 \%$ chance of slow progression, the overall lifetime risk of developing active TB is slightly less than $10 \%$. ${ }^{c} \mathrm{~TB}$ treatments last 6 to 12 months, but patient is noninfectious after a few weeks. We assume 4 weeks of infectiousness on average to account for a short period before detection. ${ }^{d} \mu_{T}=0.32$ is used for Ghana. Due to HIV/TB co-infection, it is quite reasonable to assume a larger mortality rate for TB in Ghana.

\begin{tabular}{|c|c|c|c|c|c|}
\hline \multirow[t]{2}{*}{ Parameter } & \multirow[t]{2}{*}{ Symbol } & \multicolumn{3}{|c|}{ Range of values } & \multirow[t]{2}{*}{ Reference } \\
\hline & & Min & Mode & Max & \\
\hline Probability of fast progression & $p$ & & 0.05 & & {$[9,20,28-30]^{a}$} \\
\hline Lifetime probability of slow progression & $\nu$ & & 0.00075 & & {$[20,26-28]^{b}$} \\
\hline Average duration of treatment for active TB & $1 / \gamma_{1}$ & & 0.0769 & & {$[31]^{c}$} \\
\hline Natural cure rate for active TB & $\gamma_{2}$ & & 0.058 & & {$[29,30]$} \\
\hline Mortality rate due to $\mathrm{TB}$ & $\mu_{T}$ & & $0.12^{d}$ & & {$[29,30]$} \\
\hline Proportion of immigrants that are vaccinated & $\phi$ & & 0.80 & & {$[2]$} \\
\hline Proportion of immigrants with LTBI & $\psi$ & & 0.30 & & {$[20]$} \\
\hline Average duration of vaccine protection & $1 / \omega$ & 10 & 15 & 55 & {$[32,33]$} \\
\hline Efficacy of vaccine in preventing initial infection & $q_{1}$ & 0 & & 0.50 & assumption \\
\hline Efficacy of vaccine in preventing fast progression & $q_{2}$ & 0 & & 0.50 & assumption \\
\hline Efficacy of vaccine in preventing slow progression & $q_{3}$ & 0 & & 0.50 & assumption \\
\hline Factor of protection due to latent infection & $\theta_{1}$ & 0.50 & 0.60 & 0.75 & {$[8,34]$} \\
\hline Factor of protection/susceptibility & & & & & \\
\hline due to previous active infection & $\theta_{2}$ & 0.80 & 1.00 & 1.20 & assumption \\
\hline
\end{tabular}

TABLE 2. Country-specific initial conditions, parameter values and probability density functions. $1 / \mu=$ average life expectancy (years), $\pi=$ overall recruitment rate (millions of people/year), $\alpha=$ proportion of recruitment due to immigration, LTBI $=$ proportion of the initial population with LTBI, $d=$ detection rate for active TB, $s=$ treatment success rate for active TB, $c=$ vaccination coverage, $\beta=$ transmission coefficient, $r=$ proportion of LTBI detected and treated successfully. Ranges indicate that a parameter was sampled from a uniform distribution on the particular range. ${ }^{a}$ Derived by fitting population curve to data from [6]. ${ }^{b}$ Negative value indicates a net emigration. ${ }^{c}$ Used reported coverage from 2006.

\begin{tabular}{lccccccccc}
\hline Country & \multicolumn{9}{c}{ Parameter } \\
& $1 / \mu$ & $\pi$ & $\alpha$ & LTBI & $d$ & $s$ & $c$ & $\beta$ & $r$ \\
\hline US & 78.00 & 6.416 & .22 & .074 & {$[.95,1.00]$} & {$[.60, .85]$} & .00 & {$[10,12]$} & {$[.10, .40]$} \\
UK & 78.70 & 0.9484 & .18 & .121 & {$[.78, .98]$} & {$[.60, .80]$} & .00 & {$[7,9]$} & {$[.10, .40]$} \\
GER & 78.95 & 1.092 & .25 & .083 & {$[.75, .95]$} & {$[.60, .80]$} & .00 & {$[7,9]$} & {$[.10, .40]$} \\
IND & 68.59 & 33.17 & -.01 & .62 & {$[.57, .77]$} & {$[.55, .75]$} & .74 & {$[8,10]$} & {$[.00, .30]$} \\
ROM & 71.91 & 0.3129 & -.14 & .57 & {$[.55, .95]$} & {$[.70, .90]$} & .99 & {$[17,19]$} & {$[.00, .30]$} \\
GHA & 59.12 & 0.8094 & .00 & .67 & {$[.15, .35]$} & {$[.52, .72]$} & .92 & {$[14,16]$} & {$[.00, .30]$} \\
MEX & 75.63 & 2.620 & -.22 & .237 & {$[.55, .75]$} & {$[.65, .85]$} & .99 & {$[10,13]$} & {$[.00, .30]$} \\
BRA & 72.24 & 4.616 & -.01 & .42 & {$[.55, .75]$} & {$[.60, .85]$} & .99 & {$[15,17]$} & {$[.00, .30]$} \\
\hline Source & {$[6]$} & fit $^{a}$ & {$[6]^{b}$} & fit & {$[4]$} & {$[4]$} & {$[5]^{c}$} & fit & assumption \\
\hline
\end{tabular}


TABLE 3. Results of the calibration process. Phase 0 consists of the widest reasonable ranges for parameters and were sampled from either uniform distributions or triangular distributions. All restrictions throughout the calibration process were sampled using uniform distributions. L denotes the initial proportion of the population with LTBI. The iterative process for the United States is described in detail in Figures A1-A6.

\begin{tabular}{lllllllllll}
\hline & Phase 1 & Phase 2 & Phase 3 & Phase 4 & Phase 5 & Phase 6 & Phase 7 & Phase 8 & Phase 9 & Phase 10 \\
\hline US & $\mathrm{L}[.05, .10]$ & $\beta[9,12]$ & $\mathrm{L}[.07, .075]$ & $q_{3}[.45, .55]$ & $\beta=11.3$ & $r[.45, .50]$ & $\mathrm{L}=.074$ & $\theta_{1}[.80, .90]$ & $r=.50$ & $q_{3}=.52$ \\
UK & $\mathrm{L}[.05, .10]$ & $\beta[6,9]$ & $\mathrm{L}[.12, .125]$ & $q_{3}[.30, .40]$ & $\beta=7.65$ & $\theta_{1}[.50, .60]$ & $r[0, .05]$ & $\mathrm{L}=.121$ & $q_{3}=.34$ & $q_{2}[0, .10]$ \\
GER & $\mathrm{L}[.05, .10]$ & $\beta[6,9]$ & $\mathrm{L}[.08, .085]$ & $q_{3}[.45, .55]$ & $\beta=7.95$ & $r[.45, .50]$ & $\theta_{1}[.90,1.0]$ & $r=.50$ & $q_{3}=.54$ & $\mathrm{~L}=.083$ \\
$\mathrm{IND}$ & $\beta[7,10]$ & $\theta_{1}[.60, .70]$ & $\mathrm{L}[.60, .65]$ & $q_{1}[.40, .50]$ & $\beta=8.4$ & $q_{3}[.20, .30]$ & $q_{2}[.25, .35]$ & $\theta_{1}=.75$ & - & - \\
ROM & $\beta[17,20]$ & $\theta_{1}[.60 . .70]$ & $q_{1}[.30, .40]$ & $\mathrm{L}[.55, .60]$ & $q_{3}[.30, .40]$ & $q_{2}[.20, .30]$ & $\beta=18.4$ & $\theta_{1}=.645$ & - & - \\
GHA & $\beta[14,17]$ & $\theta_{1}[.60 . .70]$ & $\mathrm{L}[.65, .70]$ & $q_{2}[.40, .50]$ & $q_{1}[.25, .35]$ & $q_{3}[.20, .30]$ & $\beta=15.1$ & $\theta_{1}=.655$ & $q_{2}=.44$ & $q_{3}=.25$ \\
MEX & $\beta[6,9]$ & $\mathrm{L}[.30, .35]$ & $q_{3}[.20, .30]$ & $q_{2}[.55, .65]$ & $\theta_{1}[.70, .80]$ & $q_{1}[.30, .40]$ & $\beta=7.5$ & $\mathrm{~L}=.33$ & $q_{3}=.24$ & - \\
BRA & $\beta[15,18]$ & $q_{2}[.10, .20]$ & $q_{1}[.25, .35]$ & $\theta_{1}[.50, .60]$ & $q_{3}[.30, .40]$ & $\mathrm{L}[.40, .45]$ & $\beta=16.1$ & - & - & - \\
\hline
\end{tabular}

\subsection{Initial conditions}

While initial disease prevalence is dictated by real data, the majority of initial conditions for the model are unknown. This includes the initial proportions of the population that are susceptible, latently infected or recovered as well as the proportions of each that are vaccinated versus unvaccinated. Even for the initial amount of active disease, we must decide the proportion to assign to the treated and untreated classes. To identify reasonable initial conditions, the model was studied using biologically reasonable parameter distributions. In general, initial conditions were sought that appeared to be near equilibrium with the motivation being that values that are consistent with real data should not display drastic variations in the initial years of simulation. It was observed that the model's dynamics were largely insensitive to initial conditions with the exception of the initial proportion of the population with latent infection. For this reason, the initial amount of latent infection in the population was included as a variable in the formal calibration procedure.

\subsection{Calibration process}

The main tool in our fitting process is what we refer to as an "adaptive simulation." An adaptive simulation allows us to compare the dynamics of our mathematical model with country-specific tuberculosis data reported by WHO (see Tables A2 and A1). Each simulation begins by using the WHO-reported prevalence as the initial amount of total active tuberculosis in the population. The simulation progresses by using the year-specific detection and treatment rates (Table A2) for active TB reported by WHO for each calendar year in the simulation. The resulting dynamics from the simulation can then be compared with the reported incidence and prevalence of active TB for the given country (see Table A1).

For each country, the process begins by using Latin Hypercube Sampling (LHS) to sample model parameters from wide, yet biologically reasonable, ranges with the goal of narrowing these ranges to those that are most consistent with reported data. Using WHO-reported detection and treatment rates for active TB ( $d$ and $s$ ), an adaptive simulation was conducted for each of 5000 parameter samplings. For each simulation, the error between simulated and reported values of disease incidence and prevalence was recorded. Error was calculated by squaring the difference between simulated and reported values for both prevalence and incidence and summing over all years in the simulation. Boxplots were constructed of this error for each parameter value. An appropriate range was then established for the parameter which exhibited the clearest influence on observed error. With this parameter restricted, a new sampling via LHS was conducted and the next most influential parameter was found. This iterative process is illustrated for the United States in Figures A1-A4. It is important to note that this process does not aim to find the parameter values which achieve a "best fit" to real data, but rather the parameter ranges and values most consistent with real data. We believe this approach to be more informative as our model contains many parameters for which reliable estimates do not exist. 
Once the process is complete, 1000 random samples of parameters are taken from the restricted parameter ranges. Simulations of the parameter samples which result in the smallest observed error for each country are depicted in Figure 2.

\subsection{Insights from the calibration process}

Beyond calibration, several insights into tuberculosis dynamics were gained from this process. One of the most striking is the epidemiological uniqueness of TB in Romania. Even when considering the full range of biologically feasible parameter values, the model performed poorly in reproducing reported data. However, a modified version of the model in which untreated active TB is detected at a rate of $d / 5$ rather than $d / 2$ (i.e. flow from $I_{T^{c}}$ to $I_{T}$ is $d / 5$ ) performed admirably. This seems to indicate an inconsistency in TB data which is confirmed by the fact that Romania's reported detection and treatment rates for active TB $(d$ and $s)$ are more consistent with those of countries with low TB burden, yet Romania's TB burden remains quite high (see Tables A2 and A1).

The iterative process of determining which parameters are most influential in allowing the model to match data also provides key insight (see Table 3). One pattern which emerges involves the importance of the initial proportion of the population with LTBI. We can see that LTBI is a more dominant factor in low incidence countries (Germany, UK, US) as it was initially the variable which most influenced observed error and is refined several times throughout the process. For higher incidence countries, LTBI is not considered until later phases. Conversely, the factor of susceptibility to exogenous reinfection, $\theta_{1}$, is much more influential in the high incidence setting. As one would expect, we see that the vaccine efficacies are, in general, more prominent in high incidence countries. However, the efficacy of vaccination in preventing slow progression to active TB $\left(q_{3}\right)$ is quite important in low incidence countries. This results from the fact that immigrants, typically from countries that mass vaccinate, play a large role in the TB dynamics of their new country.

In general, the model exhibits a good fit with data for the eight countries in our study (see Figure 2). As we might expect with a compartmental ODE model, the fits for high incidence countries tend to be better than those of low incidence countries. This is due to the fact that the mathematical formulation of our model assumes homogeneity among individuals, an assumption which is much more restrictive in the low incidence setting where individuality and spatiality may play a much larger role. The fit for Germany (see Figure 2) is the most unsatisfactory in the study. However, this may be attributed to the fact that reported treatment success rates ( $s$ in Table A2) are considerably lower for the first three years of simulation than the remainder. This change has a noticeable impact on the adaptive simulation, but such an impact is not observed in the reported incidence and prevalence. Although the fit for India is admirable, an oddity in the reported data is worth mentioning. The prevalence of active TB in India has decreased over the decade from 1995 to 2005 from 533 to 299 per 100000 population, but the incidence for every year in this period has been 168 per 100000 population (see Table A1). Beyond being completely improbable, this anomaly may have contributed to the fact that the transmission coefficient, $\beta$, which resulted from the calibration process for India, is more consistent with those of low incidence countries (see Table 3).

Another issue worth mentioning is that all of the parameters which dictate the length of active infection (i.e. $\mu, \mu_{T}, \gamma_{1}, \gamma_{2}, d$ and $s$ ) have either been previously fixed or are prescribed during the course of an adaptive simulation. Hence, the calibration process has no capacity to manipulate this quantity. Following [35], one method of estimating the average duration of an active infection is to divide disease prevalence by disease incidence. Remarkably, many of our adaptive simulations approximate this ratio well despite the inability of the calibration process to adjust this quantity.

Lastly, the resulting estimates for the initial proportion of the population with LTBI and the transmission coefficient $\beta$ should be examined more closely. These are the main products of our fitting process as all other parameters are sampled from ranges determined by biological intuition or the tuberculosis literature in our numerical investigations. Hence, it is appropriate to compare the estimates which emerged from our calibration process with the limited existing estimates. Under the formulation of the 

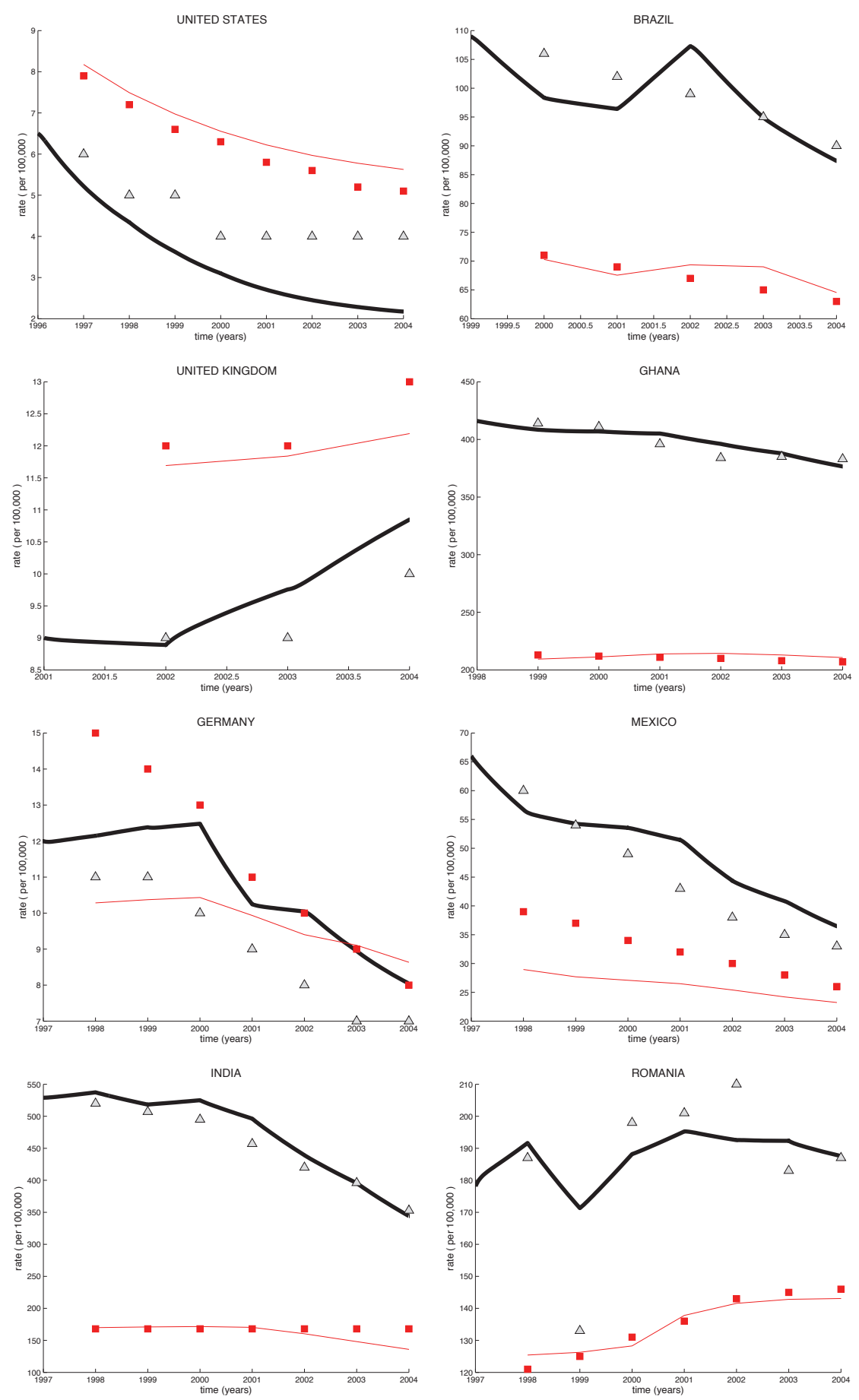

FiguRE 2. Plots of incidence (thin red) and prevalence (thick black) of active TB in the United States, the United Kingdom, Germany and India. Squares represent incidence reported by WHO. Triangles represent prevalence reported by WHO. The simulations are adaptive in that they use WHO-reported detection and treatment success rates $(d$ and $s$ ) for each calendar year. All other parameters are fixed throughout the simulation. 
model, $\beta$ gives the average number of people per year that an actively infected individual will infect in a completely susceptible population. The World Health Organization states that an actively infected individual will infect between 10 and 15 people per year on average if left untreated [36]. In Table 3, we see that the restricted ranges for $\beta$ are consistent with this assertion. In [35], the TB burden of the 22 highest-incidence countries are estimated, including the prevalence of LTBI. Both Brazil and India are included in this study. The estimates for LTBI are considerably lower than those reached in our calibration process (25\% versus $42 \%$ for Brazil and $44 \%$ versus $62 \%$ for India). The estimate for LTBI in the United States of $7.4 \%$ is also higher than the $4.2 \%$ LTBI prevalence estimated in [37].

\section{When is vaccination the best policy?}

In studying the tradeoff between vaccination and detection/treatment of LTBI, we hope to gain an understanding of when the discontinuation of mass vaccination with BCG is or is not justified. Here, we determine the rates of LTBI treatment necessary to prevent as many cases of active TB as mass vaccination with BCG. For illustration, we let each vaccine efficacy and detection/treatment range from 0-100\% and compare the cumulative number of new active TB infections for scenarios with vaccination of $98 \%$ and $0 \%$ of newborns. Figure 3 shows the results of this experiment with blue dots (red boxes) indicating points in which vaccination results in a lower (higher) cumulative incidence of active TB.

It is apparent that over 20 years mass vaccination will very likely be beneficial for any country as seen in the large ratio of blue dots to red squares. Another pattern that emerges and is illustrated in Figure 3, is that countries with higher TB burden (i.e. Brazil, Ghana, India, Mexico, Romania) have a larger proportion of sample parameters in which mass vaccination is the better policy (more blue dots than low incidence countries), while low burden countries (i.e. Germany, UK, US) have a larger proportion of samples in which not vaccinating results in fewer cases of active TB (more red boxes than high incidence countries). This relationship is in good agreement with the WHO recommendation of universal vaccination for high incidence countries. To reinforce the observation, we note that the sampling of LTBI detection/treatment rates from values of $0-100 \%$ was done for illustration, whereas realistic will be significantly smaller in high incidence countries than those in low incidence ones.

In general, the following relationship emerges for low incidences countries (see Figure 3): assuming vaccination protected against initial infection only, replacing a mass vaccination policy by a no vaccination policy will result in increased TB incidence unless a country is able to detect and treat a proportion of the latently infected class that is approximately equal to twice the vaccine's efficacy; for a vaccine protecting against slow progression only, LTBI must be detected and treated on a proportion about equal to the vaccine's efficacy in order for vaccine discontinuation to lead to reduced TB levels; when the vaccine prevents against fast progression to active TB, the threshold lies between the previous two.

Further examination shows that reinfection is responsible for the differences between low and high incidence countries. As treatment of LTBI moves individuals into the recovered class, they become exposed to reinfection. Hence, larger reinfection rates after recovery will render treatment of LTBI less effective at reducing TB incidence. On the other hand, vaccination provides no direct protection against exogenous reinfection whereas treatment of LTBI can eliminate the chance of exogenous reinfection for some individuals.

In Figure 4, we see the effect of omitting exogenous reinfection and reinfection after recovery. Comparing the graphs for the US, UK and Germany in Figures 3 and 4, we see that low levels of active disease render reinfection insignificant in these low burden settings. While omitting both forms of reinfection is not indicative of reality, the fact that doing so produces thresholds for low and high incidence countries that agree quite closely (see Figure 4) shows that reinfection is the driving force behind the differences between low and high incidence countries. 

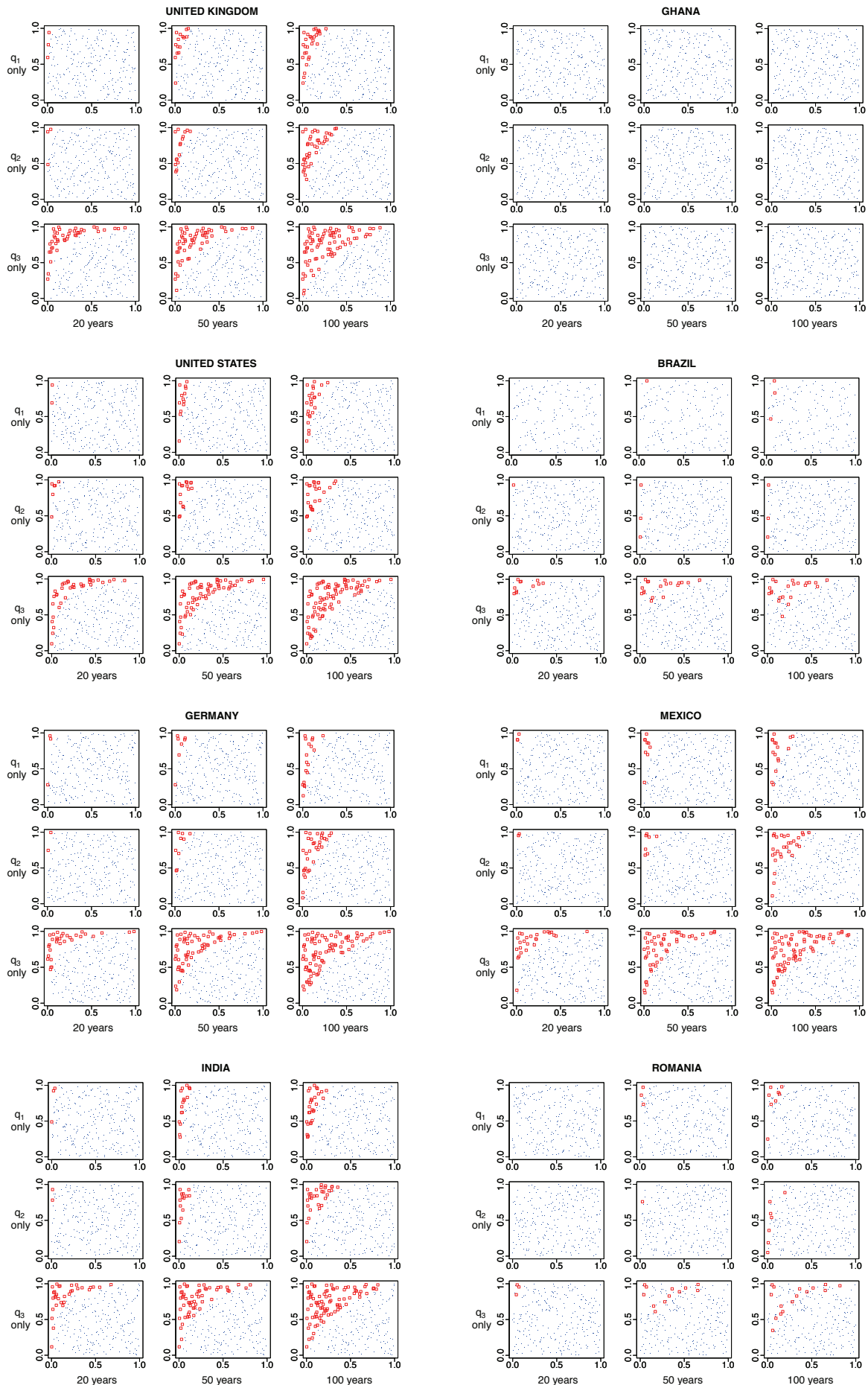

FIGURE 3. Regions of parameter space where vaccination is (or is not) the better strategy when exogenous reinfection of LTBI and infection after recovery are considered (i.e. $\theta_{1}$ sampled from 0.50 to 0.75 and $\theta_{2}$ sampled from 0.80 to 1.20). A blue dot (red box) indicates a parameter sample in which vaccination results in a lower (higher) incidence of active TB. Each row corresponds to one mode of vaccine protection $\left(q_{1}, q_{2}, q_{3}\right)$. The other efficacies are set to 0 . The columns give results for 20,50 and 100 years. 

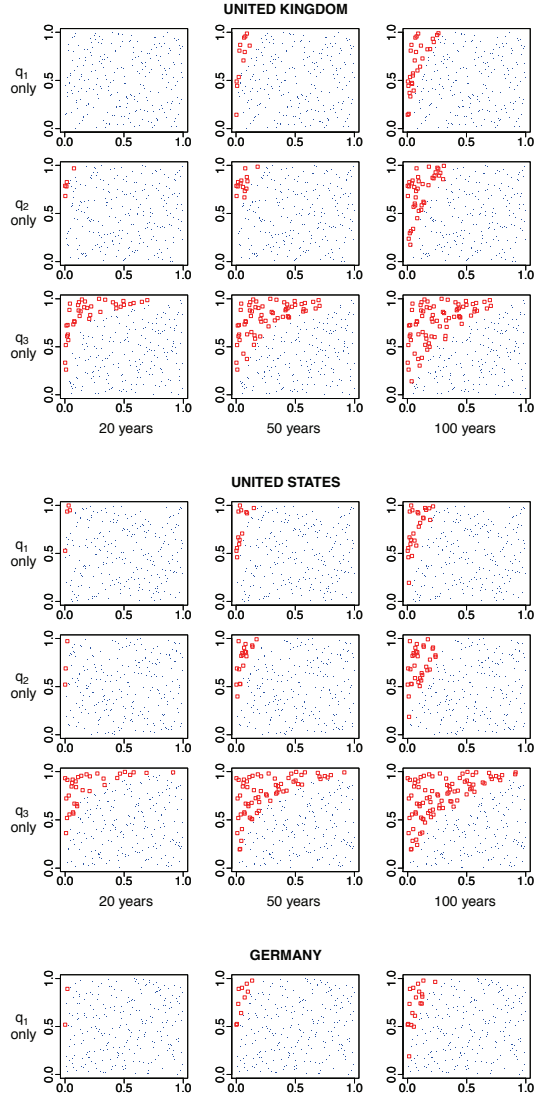

GERMANY
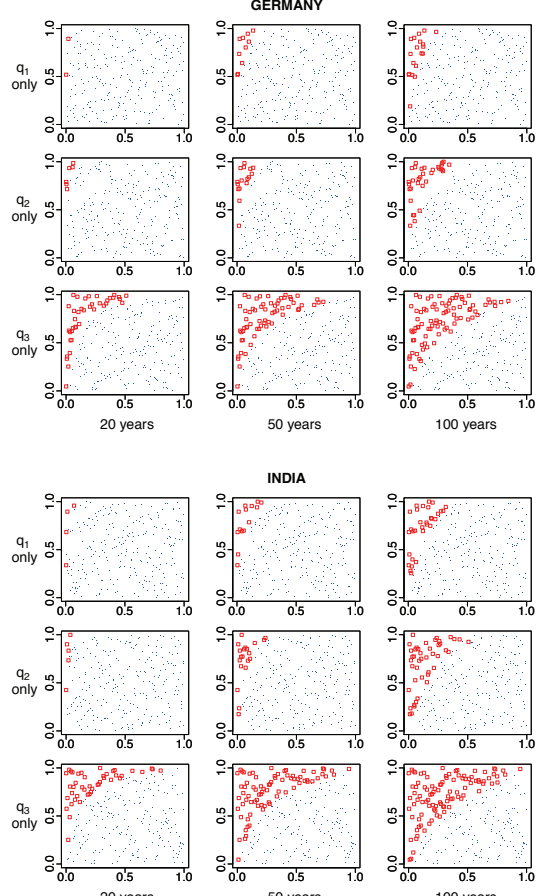
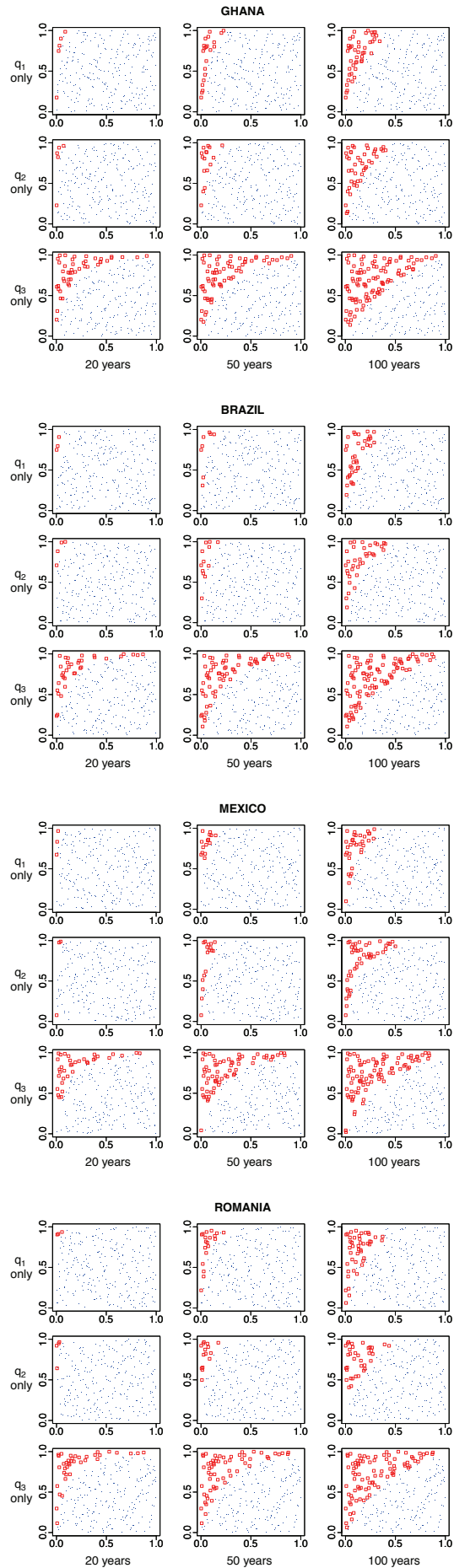

FIGURE 4. Regions of parameter space where vaccination is (or is not) the better strategy when exogenous reinfection of LTBI and infection after recovery are omitted (i.e. $\theta_{1}=$ $\theta_{2}=0$ ). A blue dot (red box) indicates a parameter sample in which vaccination results in a lower (higher) incidence of active TB. Each row corresponds to one mode of vaccine protection $\left(q_{1}, q_{2}, q_{3}\right)$. The other efficacies are set to 0 . The columns give results for 20, 50 and 100 years. 

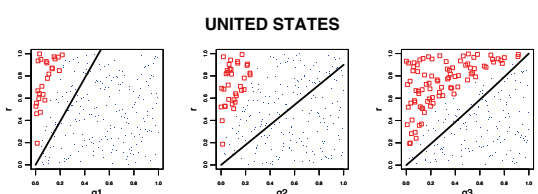

GERMANY
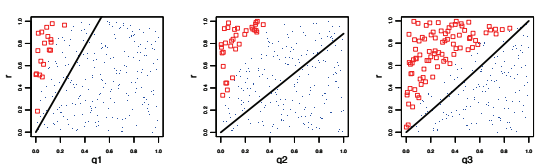

ROMANIA
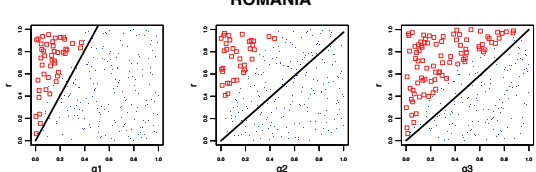

MEXICO
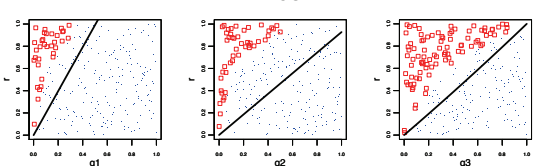
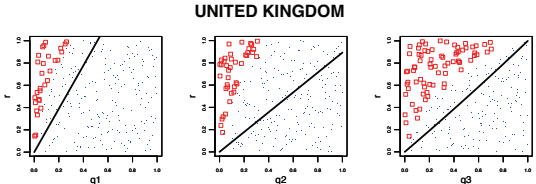

INDIA
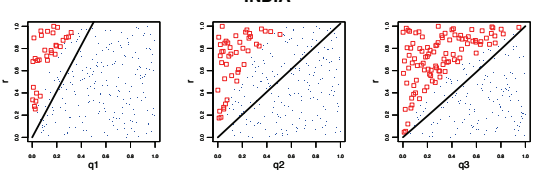

GHANA
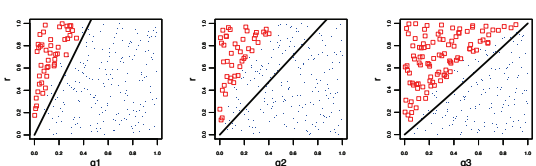

BRAZIL
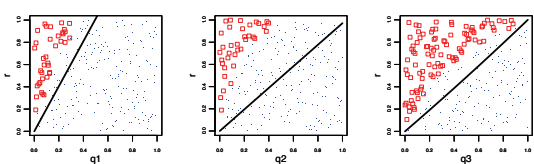

FiguRE 5. Comparison of analytic thresholds obtained via the basic reproduction number in [25] versus patterns observed in this study using recent TB indicators. Analytic thresholds are depicted by solid black lines. The scatterplots depict results after 100 years when exogenous reinfection is neglected (i.e. $\theta_{1}=0$ ).

\subsection{Comparison between numerical study and theoretical thresholds}

Using stability analysis of the disease-free equilibrium and the basic reproductive number, analytic thresholds are established in [25] that dictate the proper vaccination policy for a society at the brink of eradicating tuberculosis. The basic reproductive number gives the average number of secondary infections caused by a single infectious individual introduced into a completely susceptible society.

When each mode of protection conferred by vaccination is considered separately, the resulting thresholds are

$$
r_{q_{1}}^{*}=\frac{q_{1}(\nu+\mu p)}{\nu(1-p)}, \quad r_{q_{2}}^{*}=\frac{q_{2} \mu p}{\nu(1-p)}, \quad r_{q_{3}}^{*}=\frac{q_{3} \mu}{\mu+\nu\left(1-q_{3}\right)} .
$$

For example, if a country is only able to detect and successfully treat LTBI at a rate below $r_{q_{1}}^{*}$, then vaccination with an efficacy of $q_{1}$ at preventing initial infection will result in a smaller basic reproductive number. Hence, $r<r^{*}$ implies that vaccination is the better policy for the given society. If $r>r^{*}$, the society will be better served by not vaccinating and taking full advantage of its capacity to detect and treat LTBI.

In Figure 5, we compare the analytic thresholds with the results obtained from our numerical study. We emphasize that the analytic thresholds where obtained via the basic reproductive number, which considers the situation of one infected individual introduced into a completely susceptible population. Clearly, this assumption is not applicable to even the lowest incidence countries in the numerical study. Regardless, the behavior of the system with current TB indicators displays considerable agreement with the analytic thresholds (especially $q_{1}$ and $q_{3}$ ) when exogenous reinfection is neglected.

\section{How much better would one vaccination policy be?}

So far we have only investigated the parameter regions in which a policy of universal vaccination with BCG will or will not result in fewer cases of active TB than a policy emphasizing the ability to detect 


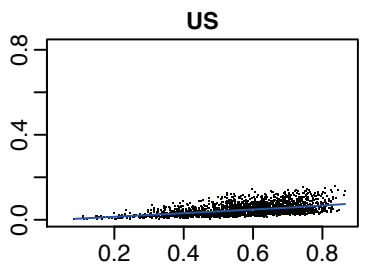

MEXICO

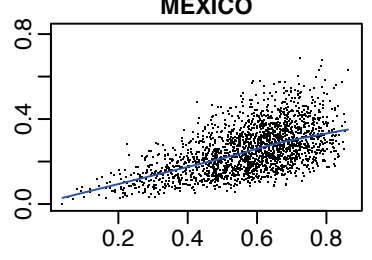

ROMANIA

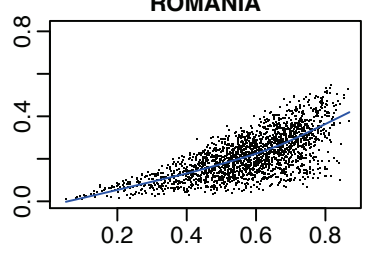

UK

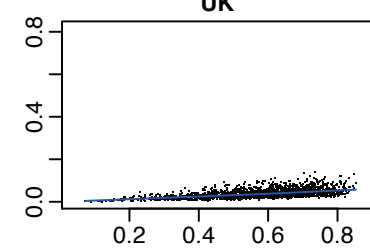

INDIA

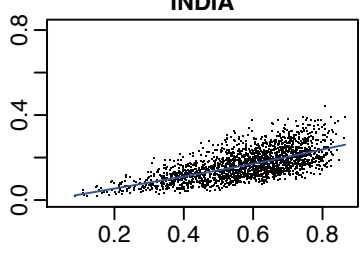

GHANA

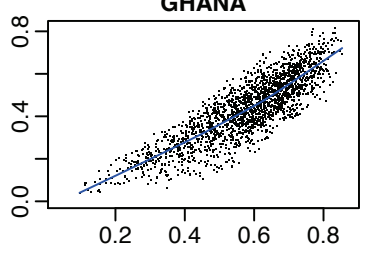

GERMANY

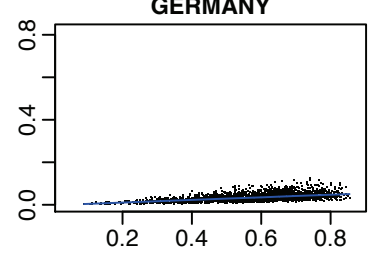

BRAZIL

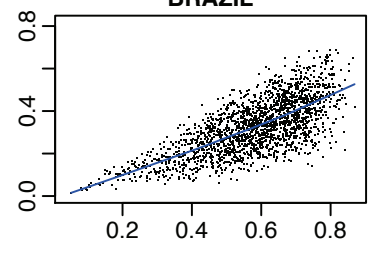

Figure 6. Cumulative proportion of active TB cases prevented by vaccination after 50 years (Horizontal axes: overall efficacy of BCG (i.e. $\left.1-\left(1-q_{1}\right)\left(1-q_{2}\right)\left(1-q_{4}\right)\right)$; Vertical axes: proportion of new infections prevented by vaccination).

and treat LTBI. Clearly this is insufficient in the public policy arena as a program which requires the vaccination of countless individuals in order to save a handful of disease cases would never be prudent.

To quantify the difference between policy alternatives, we now calculate the proportion of cases prevented by $B C G$ by subtracting the total incidence of active $\mathrm{TB}$ with vaccination from the total incidence of active TB without vaccination and dividing the quantity by the total incidence without vaccination. We stress that the proportion of cases prevented could be negative under this definition if treatment of LTBI outperforms vaccination. For this exercise, BCG and efficacies and detection/treatment rates vary through the realistic ranges of Table 1 (i.e. overall BCG efficacy of $0-88 \%$, detection/treatment of LTBI 0-30\% and 10-40\% for high and low incidence countries, respectively).

The ranges of $0-30 \%$ and $10-40 \%$ for the detection/treatment rate of LTBI are based on the intuition that these rates should be substantially smaller than their counterparts for active TB (i.e. $r<d s$ ). In a recent large-scale study in the United States, it was found that only $25.5 \%$ of individuals with LTBI had been diagnosed and that only $13.2 \%$ had been prescribed treatment [37]. Treatment for this symptomless condition is lengthy (usually 6-12 months) and often not finished by those who initiate it. In [38], the authors use estimates of 30-64\% and 69-93\% for the percentages of treatments that are completed and the efficacy of treatment when completed, respectively. This gives a net efficacy for treatment of LTBI (including adherence and effectiveness) of about 20-60\%. Hence, it is quite likely that our estimates may be overly generous recalling that our treatment rate, $r$, represents the proportion of latent infections that are detected and successfully treated (i.e. $r \approx 13.2 \% *(20-60 \%)=2.6-7.9 \%$ ).

The simulations take the current vaccination policy of a country into account. For instance, the United States currently does not vaccinate, so the initial population contains only a small number of vaccinated individuals that represent mostly immigrants with previous vaccination. The country-specific relationship between the overall efficacy of BCG (i.e. $\left.1-\left(1-q_{1}\right)\left(1-q_{2}\right)\left(1-q_{4}\right)\right)$ and the cumulative proportion of active TB cases prevented by vaccination after 50 years is shown in Figure 6 . 
TABLE 4. Vaccinations per case of active TB prevented after 50 years

\begin{tabular}{lrrrrr}
\hline Country & \multicolumn{5}{c}{ Percentile } \\
& $5^{\text {th }}$ & $25^{\text {th }}$ & $50^{\text {th }}$ & $75^{\text {th }}$ & $95^{\text {th }}$ \\
\hline US & 1377 & 2531 & 4596 & 7615 & 14844 \\
UK & 1135 & 2084 & 3118 & 4787 & 9807 \\
Germany & 1076 & 1978 & 2980 & 4849 & 10146 \\
Mexico & 97 & 220 & 391 & 691 & 1383 \\
India & 96 & 166 & 249 & 379 & 700 \\
Brazil & 13 & 29 & 53 & 100 & 250 \\
Romania & 4 & 13 & 30 & 79 & 263 \\
Ghana & 6 & 11 & 16 & 23 & 43 \\
\hline
\end{tabular}

In accordance with prevailing views, our model illustrates that mass vaccination in low incidence countries results in proportionally fewer preventions than in high incidence countries (median preventions of $3.3-4.3 \%$ versus $16.3-44.5 \%$ after 50 years). More importantly, mass vaccination resulted in lower TB incidence than treatment of LTBI in all of the 2000 parameter samplings carried out for each of the eight countries. As the range of treatment rates for LTBI may even be optimistic, we can conclude that it is very unlikely that treatment of LTBI would outperform mass vaccination in any setting.

In Figure 6, we notice the dramatic preventive effect of vaccination in Ghana despite the fact that the sampled detection/treatment rates of LTBI (0-30\%) are actually quite similar to their counterparts for active infection (8-25\%, see Table 2) and hence are most likely large overestimates. This demonstrates the positive impact of a largely imperfect vaccine in a high incidence setting. In fact, rates of detection and treatment of LTBI in many resource-poor settings may be low enough that there really is no tradeoff for BCG vaccination in terms of LTBI detection. In such settings, the main issue is whether BCG provides enough protection to justify its widespread use. As our modeling has incorporated strict assumptions against BCG and optimistic assumptions for LTBI detection in the absence of BCG, it only provides a low estimate for the preventive capacity of BCG in such high incidence, low resource settings.

\section{When is mass vaccination justified?}

To provide insight into the economic costs of policy shifts regarding BCG, we examine the efficiency of vaccination by tracking the number of vaccinations per prevented case of active TB. Initially, this indicator is very large as time is needed for protection to build in the population. As time progresses, the number of vaccinations per prevention steadily decreases and eventually stabilizes. Table 4 shows the distribution after 50 years to balance the need to include long-term behavior with the realization that conditions will change substantially over the course of decades.

As would be expected, the simulations show that the efficiency of BCG vaccination in preventing cases of active TB is significantly reduced in countries of low TB burden. In general, the number of vaccinations necessary to prevent one case of active TB appears to be two orders of magnitude larger for low incidence countries than for most high incidence countries. Estimating the number of vaccinations required per active infection prevented is a first step in assessing both financial and non-financial costs associated with vaccination. For example, the WHO has recently revised its guidelines regarding BCG to indicate that HIV infected children (regardless of symptoms) should not be immunized with BCG due to increased risk of disseminated BCG disease [39,40]. Unfortunately, implementation of this policy change may be difficult given the lack of HIV testing in children in many countries with high HIV burdens. To quantify the ramifications of this adverse effect of BCG, one could make setting-specific estimates of the number of TB cases prevented per case of disseminated BCG disease caused by BCG vaccination using the number of BCG vaccinations per case of active TB prevented, increased risk of disseminated BCG disease [40], antenatal HIV prevalence and vertical transmission rate. 


\section{Discussion}

In this article, we have considered a mathematical model for the epidemiology of tuberculosis in order to study the trade-off that exists between the benefits of vaccination and the ability to detect and treat LTBI. The model includes vaccination, treatment of LTBI, treatment of active TB, and immigration and makes the strict assumption that vaccination with BCG renders LTBI undetectable.

Thresholds of vaccine efficacy and LTBI treatment rates were established that indicate when mass vaccination results in a reduction of tuberculosis incidence. Each mode of protection was considered separately. Assuming vaccination protected against initial infection only, replacing a mass vaccination policy by a no vaccination policy will result in increased TB incidence unless a country is able to detect and treat a proportion of the latently infected class that is approximately equal to twice the vaccine's efficacy. For a vaccine protecting against slow progression only, LTBI must be detected and treated in a proportion about equal to the vaccine's efficacy in order for vaccine discontinuation to lead to reduced TB levels. When the vaccine prevents against fast progression to active TB, the threshold lies between the previous two.

Moving to quantify the impact of a country's vaccination policy choice, the percentage of new cases of active TB prevented as a function of overall vaccine efficacy was observed. Given that vaccination outperformed detection/treatment of LTBI in all of the 2000 parameter sampling carried out for each country, we are able to conclude that it is highly unlikely that mass vaccination would ever result in increased TB incidence for any country, including those with low TB burden. This is in spite of the model's strict assumptions against BCG and optimistic assumptions regarding detection and treatment of LTBI. This conclusion suggests that the public health choice between a policy of mass vaccination and one of no vaccination in low burden countries is not really made on the basis of epidemiological considerations but rather on political/economic grounds.

To further consider whether mass vaccination is justifiable in low incidence countries, the number of vaccinations per case of active TB prevented was observed. Our results indicate that vaccination would be much less efficient in a low incidence setting than in a high incidence one. While we must be careful to not overestimate the quantitative and predictive ability of such simulation over a 50-year period, the general pattern of thousands of vaccinations per prevention in low incidence countries versus dozens in high incidence countries is noteworthy.

A discussion of whether the thousands of vaccinations needed per prevention in low incidence countries actually does or does not justify the lack of a mass vaccination policy should be based on social and economic considerations that are beyond the scope of this work. However, it seems that the continued emergence of drug-resistant and multi-drug resistant TB should only increase the number of vaccinations per prevention that would be considered acceptable for at least two important reasons. First, drug resistance renders treatment of tuberculosis a much more complicated and expensive process. Perhaps more importantly though, incomplete treatment is a major source of drug resistance and, hence, any measure that focuses on prevention rather than treatment should be considered more favorable from this perspective. What is clear from this work is that the policy choice regarding mass vaccination is much more about reinfection (both exogenous and in recovered individuals) than it is about BCG's interference with the detection of LTBI, despite this interference's prominent role in the BCG policy statements of many countries.

In the present time of economic strain, results like those found in this study beckon governments to conduct comprehensive studies that realistically assess the likely impact of their policies before implementing them. 


\section{A. Appendix}

\section{A.1. TB indicators from the World Health Organization}

The World Health Organization maintains a Global TB Database that monitors many aspect of the disease in its member countries [4]. Indicators in the database include disease incidence, disease prevalence, TBrelated deaths, reported cases, DOTS coverage, case detection rates, treatment success rates, etc. In Tables A1 and A2, we present indicators either directly from or derived from the database that are applicable to this work.

TABLE A1. Tuberculosis indicators derived from the World Health Organization's Global TB Database [4]. "Inc" denotes the incidence of active TB which is the number of new cases reported in a calendar year. "Prev" is the prevalence of active infection. Both indicators are reported as rates per 100000 population.

\begin{tabular}{llrrrrrrrrrrr}
\hline & & 1995 & 1996 & 1997 & 1998 & 1999 & 2000 & 2001 & 2002 & 2003 & 2004 & 2005 \\
\hline \multirow{2}{*}{ US } & inc & 8.5 & 7.9 & 7.2 & 6.6 & 6.3 & 5.8 & 5.6 & 5.2 & 5.1 & 4.9 & 4.7 \\
& prev & 6 & 6 & 5 & 5 & 4 & 4 & 4 & 4 & 4 & 4 & 3 \\
\hline UK & inc & 12 & 12 & 12 & 12 & 12 & 12 & 12 & 12 & 13 & 13 & 14 \\
& prev & 9 & 9 & 9 & 9 & 9 & 9 & 9 & 9 & 10 & 10 & 11 \\
\hline Germany & inc & 16 & 15 & 15 & 14 & 13 & 11 & 10 & 9 & 8 & 8 & 7 \\
& prev & 13 & 12 & 11 & 11 & 10 & 9 & 8 & 7 & 7 & 6 & 6 \\
\hline India & inc & 168 & 168 & 168 & 168 & 168 & 168 & 168 & 168 & 168 & 168 & 168 \\
& prev & 533 & 529 & 520 & 507 & 495 & 457 & 420 & 396 & 353 & 312 & 299 \\
\hline Romania & inc & 112 & 116 & 121 & 125 & 131 & 136 & 143 & 145 & 146 & 140 & 134 \\
& prev & 172 & 178 & 187 & 133 & 198 & 201 & 210 & 183 & 187 & 179 & 146 \\
\hline Ghana & inc & 217 & 216 & 214 & 213 & 212 & 211 & 210 & 208 & 207 & 206 & 205 \\
& prev & 454 & 455 & 416 & 414 & 411 & 396 & 384 & 385 & 383 & 383 & 380 \\
\hline Mexico & inc & 45 & 42 & 39 & 37 & 34 & 32 & 30 & 28 & 26 & 24 & 23 \\
& prev & 71 & 66 & 60 & 54 & 49 & 43 & 38 & 35 & 33 & 31 & 27 \\
\hline Brazil & inc & 79 & 77 & 75 & 73 & 71 & 69 & 67 & 65 & 63 & 61 & 60 \\
& prev & 120 & 117 & 113 & 109 & 106 & 102 & 99 & 95 & 90 & 81 & 76 \\
\hline
\end{tabular}

\section{A.2. Calibration process}

In Figures A1-A6, we illustrate the iterative process for calibrating the model to TB data from the World Health Organization's Global TB Database [4] for the United States. The general idea is to sample model parameters from wide, yet biologically reasonable, ranges with the goal of iteratively narrowing these ranges to those that are most consistent with reported data. This is done by conducting adaptive simulations for each sampling and restricting the range of the parameter that exhibits the clearest influence on observed error.

Acknowledgements. The authors would like to acknowledge previous institutions at which substantial portions of this research were conducted. DJG and FAM: Department of Mathematics, Purdue University, DJG: Center for Biomedical Modeling at UCLA. 

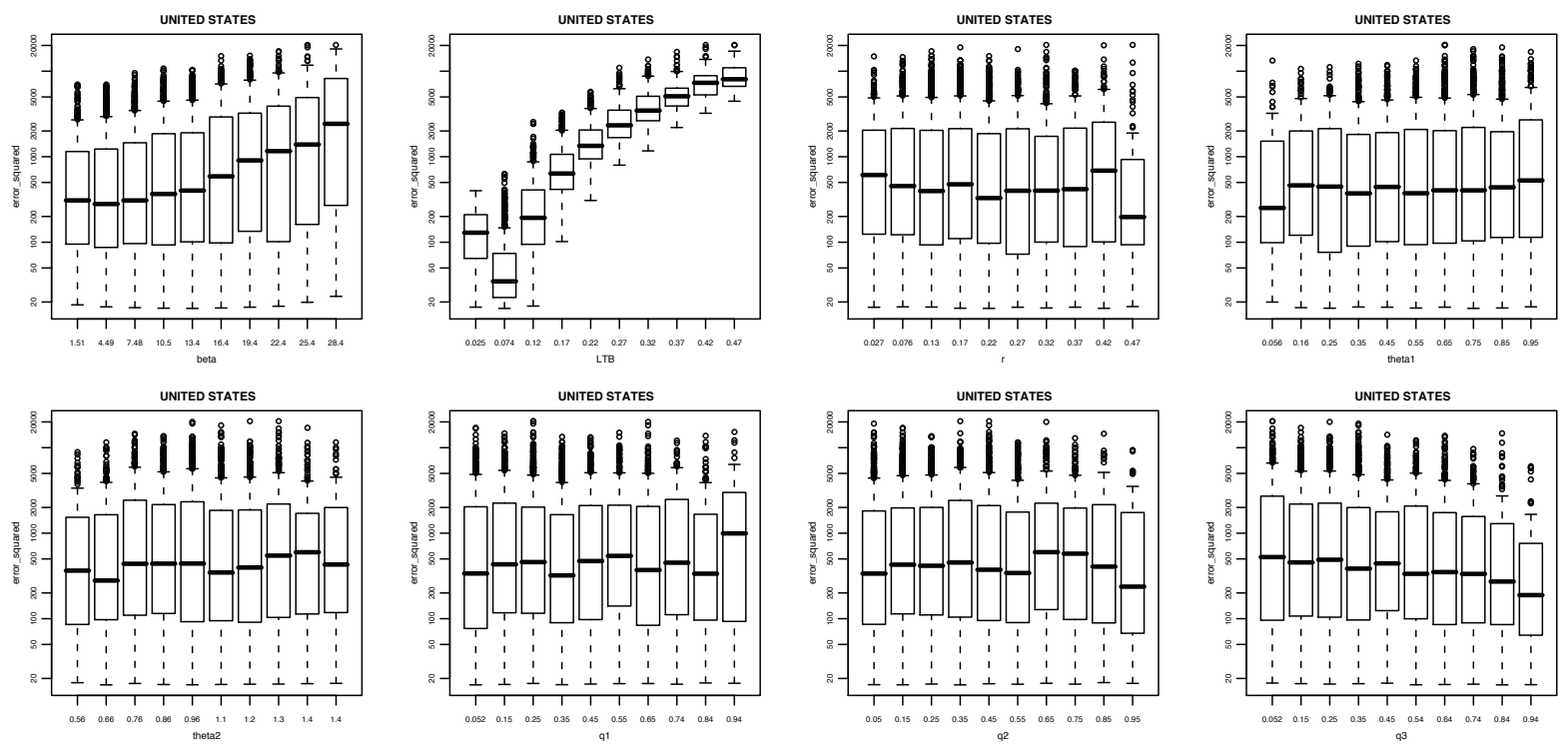

Figure A1. Phase 0: Boxplots of simulation errors for varying ranges of each parameter resulting from the initial 5000 parameter samples for the United States. It appears that the initial proportion of the population with latent TB has the clearest influence on error. The range of $[0.05,0.10]$, labeled by its midpoint, corresponds to the smallest errors. Hence, LTB is restricted to values between 0.05 and 0.10 in the next sampling (Phase 1).
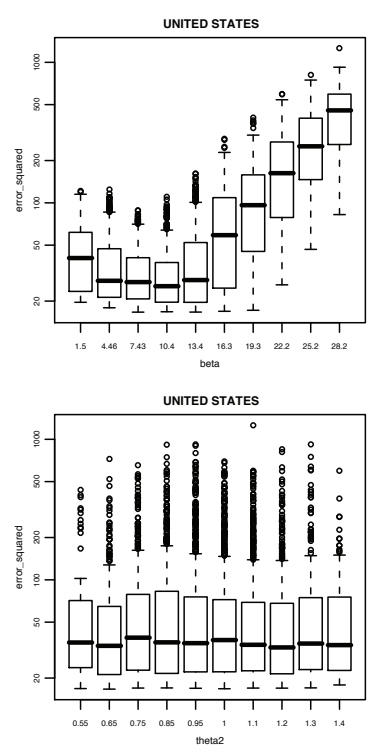
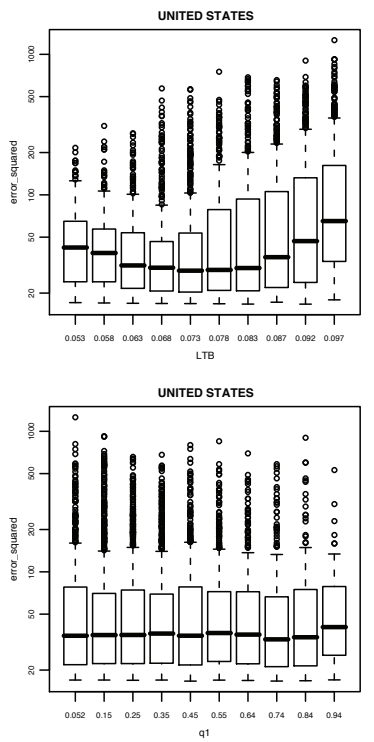
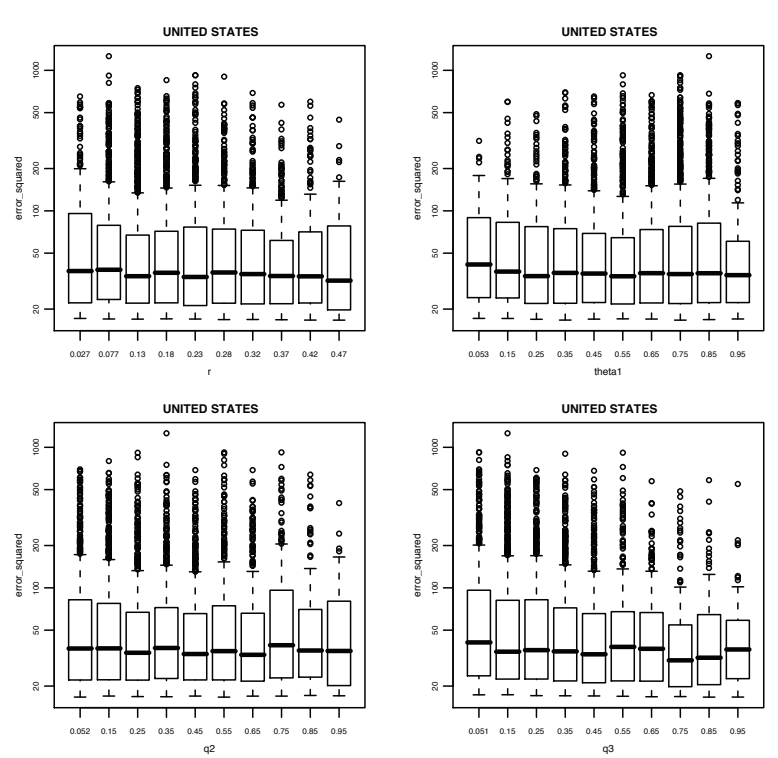

Figure A2. Phase 1: It appears that $\beta$ has the clearest influence on error. The range of $[9,12]$ seems to correspond to the smallest average errors. Hence, $\beta$ is restricted to this range in the next sampling (Phase 2). 

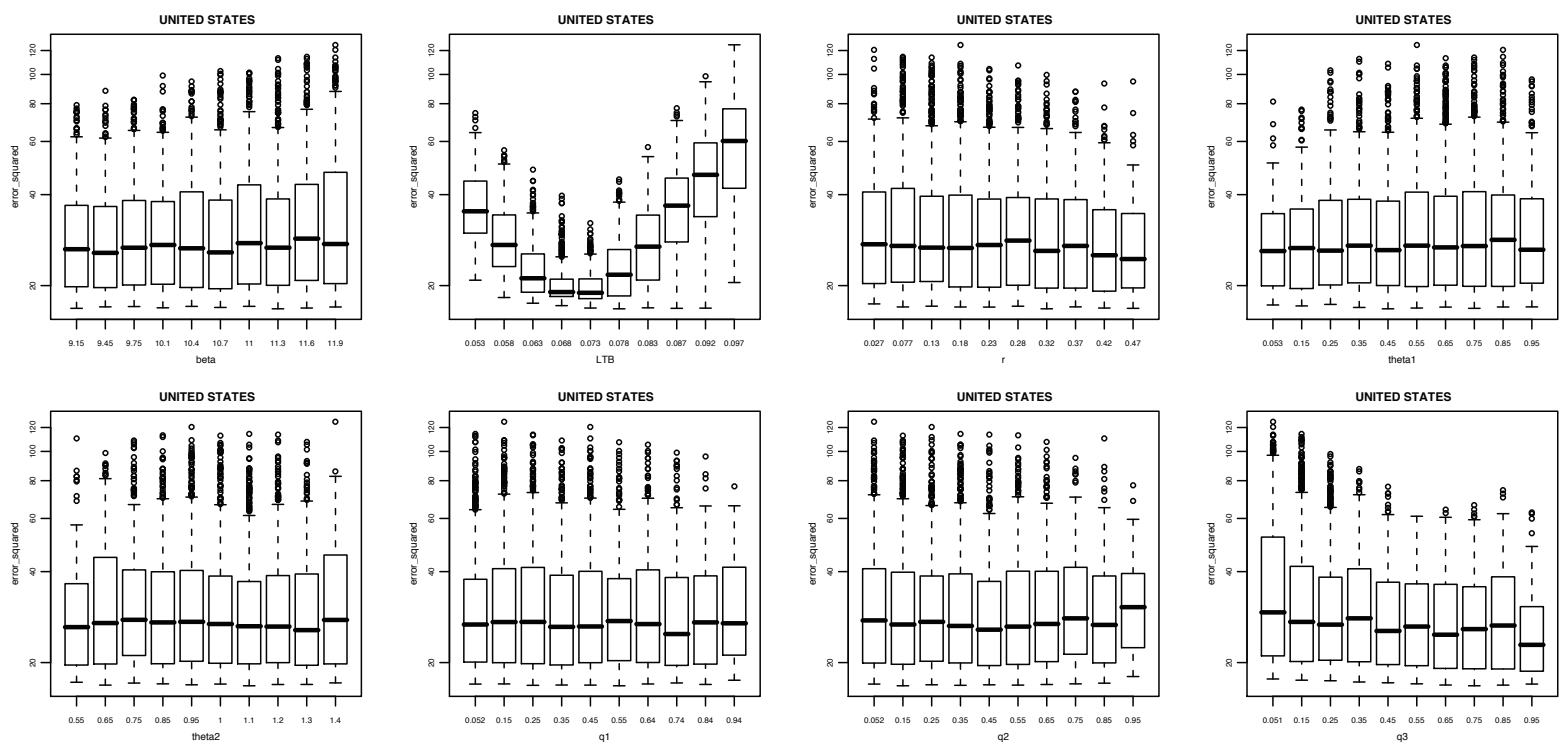

Figure A3. Phase 2: It appears that the initial proportion of the population with latent TB once again has the clearest influence on error. The range of $[0.070,0.075]$ seems to correspond to the smallest average errors. Hence, LTB is restricted to this range in the next sampling (Phase 3).
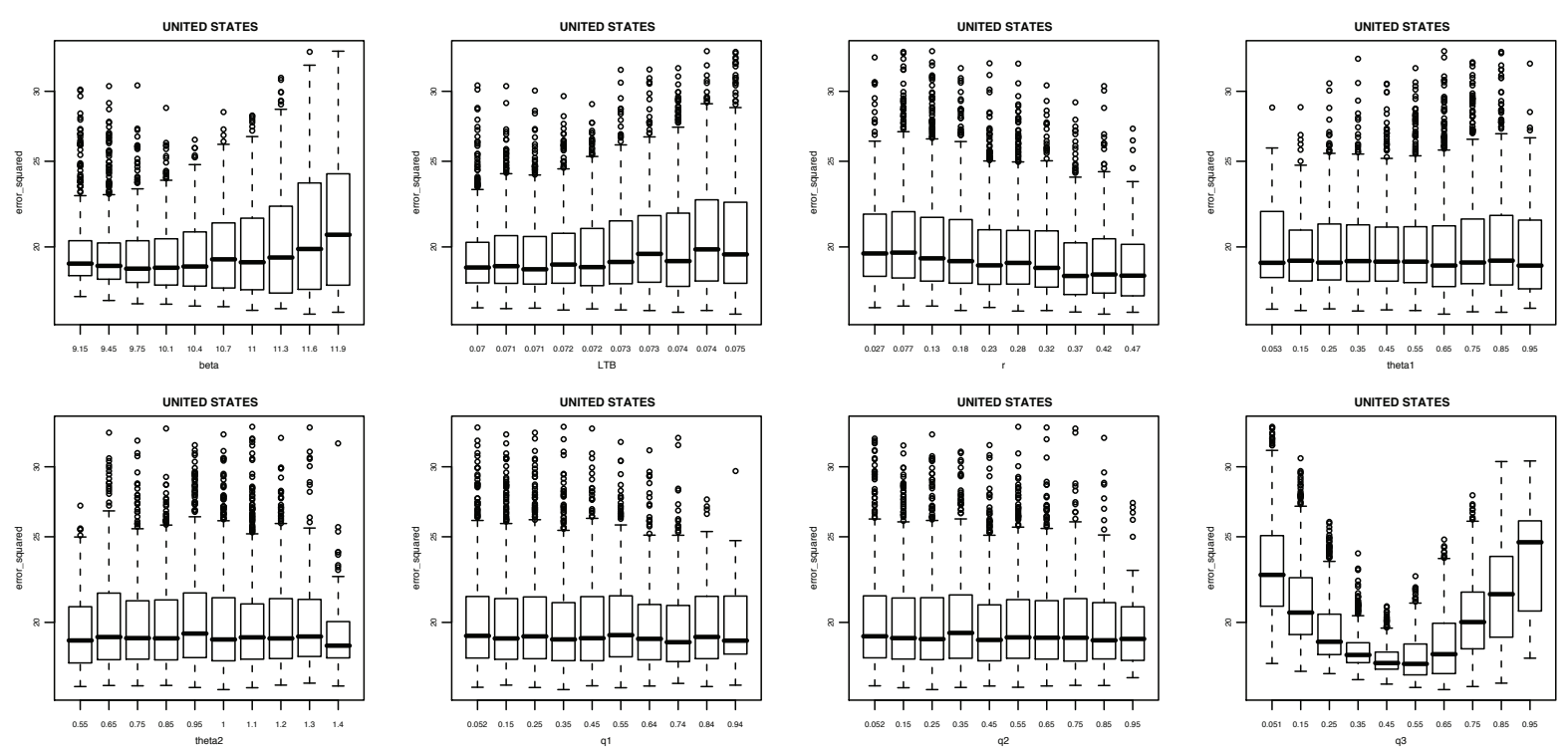

Figure A4. Phase 3: It appears that $q_{3}$ has the clearest influence on error. The range of $[0.45,0.55]$ seems to correspond to the smallest average errors. Hence, $q_{3}$ is restricted to this range in the next sampling (Phase 4). 

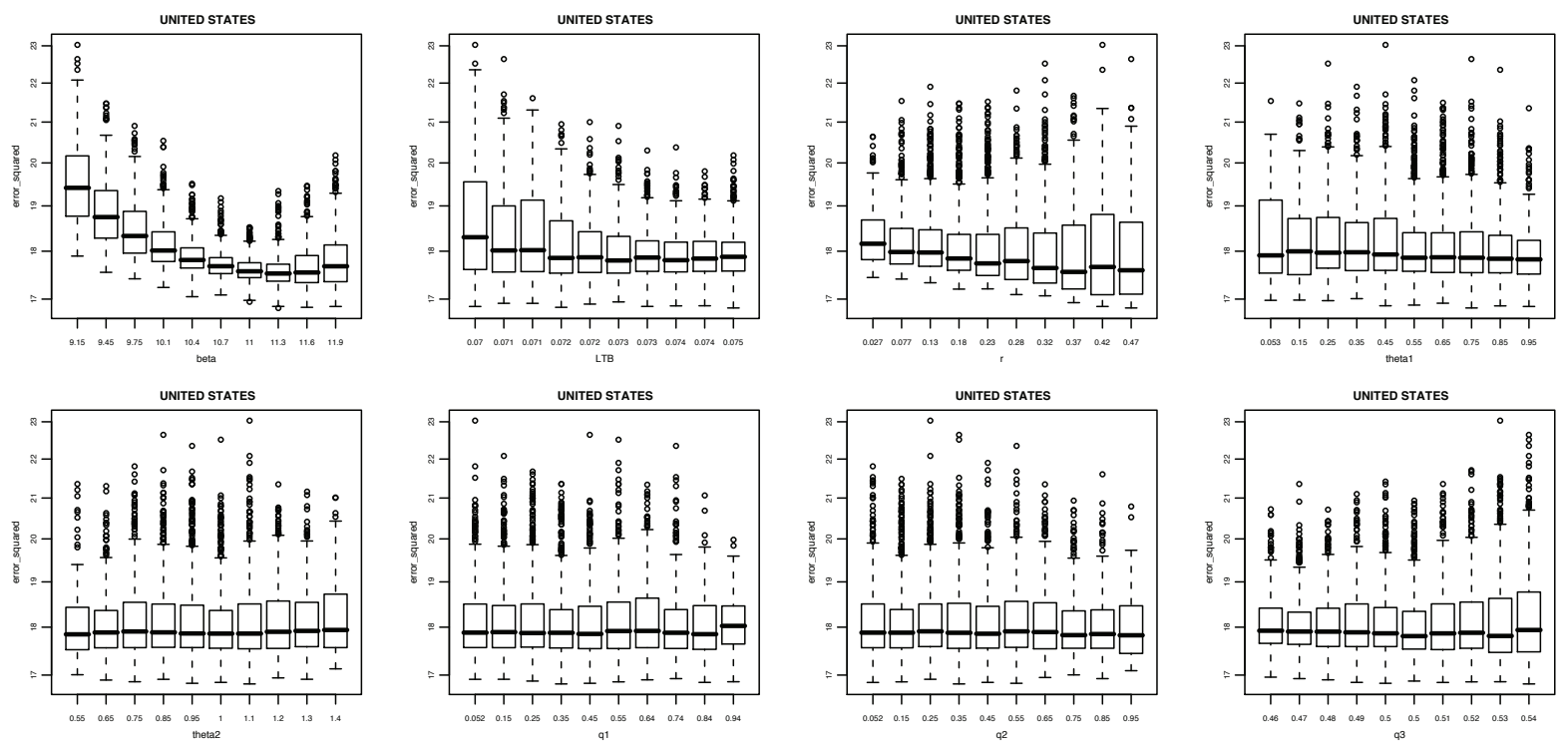

Figure A5. Phase 4: It appears that $\beta$ once again has the clearest influence on error. As $\beta$ is already quite restricted, we now fix $\beta$ at 11.3 so that we may continue the calibration process in Phase 5.
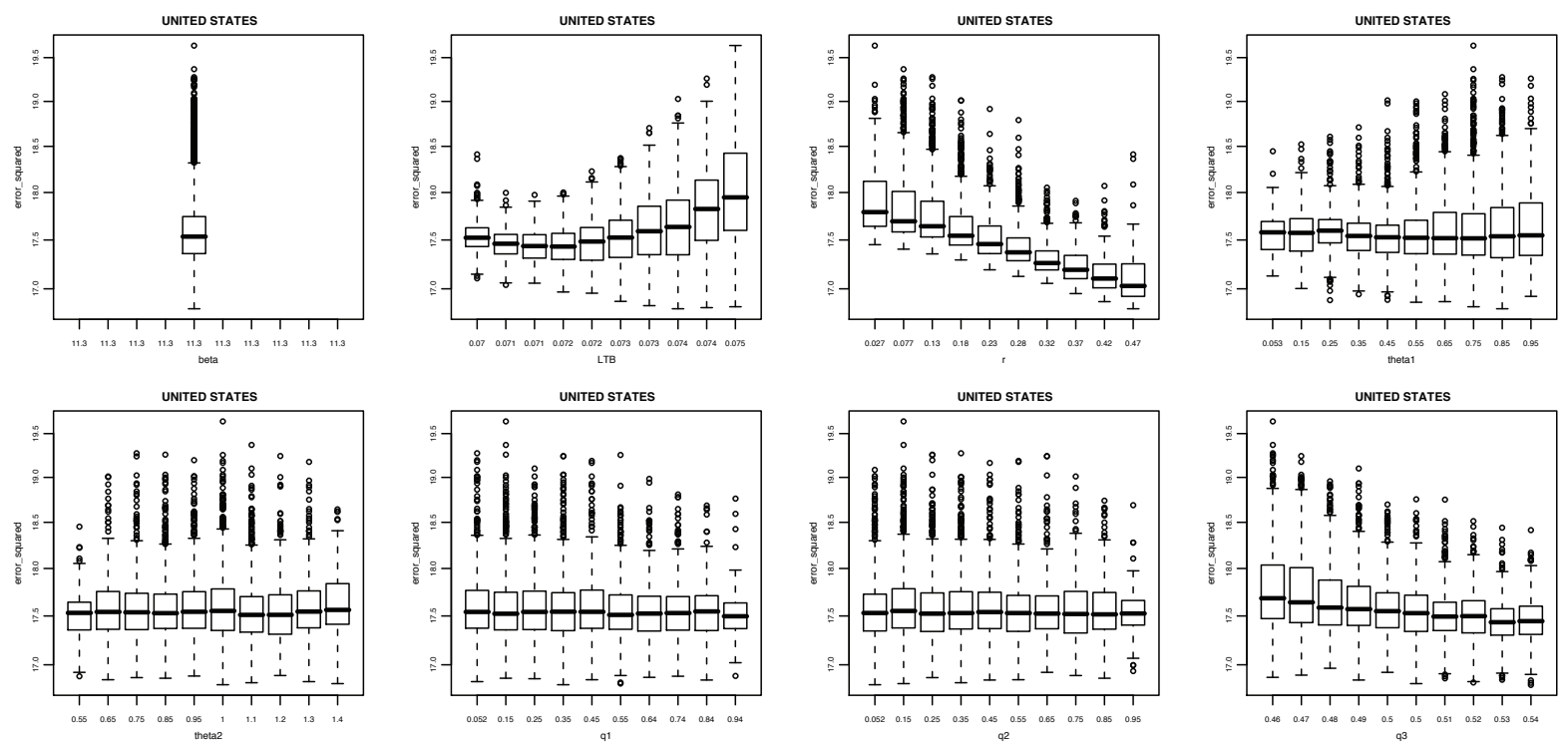

Figure A6. Phase 5: It appears that $r$ has the clearest influence on error. The range of $[0.45,0.50]$ seems to correspond to the smallest average errors. Hence, $r$ is restricted to this range in the next sampling (Phase 6). Although this calibration process is continued through ten phases, we omit the remaining phases of this process for brevity and summarize the findings of this analysis in Table 3 . 
TABLE A2. Tuberculosis indicators derived from the World Health Organization's Global TB Database [4]. In the model, $d$ denotes the detection rate for active TB. We use WHO indicator whole country all new cases detection rate. The proportion of treatments which successfully cure the patient is denoted by $s$ in the model. We calculate it by the formula $s=($ DOTS treatment success $)($ DOTS population coverage $)+($ Non-DOTS treatment success $)(1-D O T S$ population coverage). Following [41], we use WHO treatment success (defined as the proportion that are cured plus the proportion of cases that complete the full course of treatment) as a proxy for the cure rate. ${ }^{a} \mathrm{WHO}-$ reported whole country all new cases detection rate values for the US are greater than $100 \%$. We use $100 \%$ for biological feasibility. ${ }^{b}$ Value of 0.65 was assumed in order to have 4 consecutive years for simulation.

\begin{tabular}{llrrrrrrrrrrr}
\hline & & 1995 & 1996 & 1997 & 1998 & 1999 & 2000 & 2001 & 2002 & 2003 & 2004 & 2005 \\
\hline \multirow{2}{*}{ US } & $d^{a}$ & 1.00 & 1.00 & 1.00 & 1.00 & 1.00 & 1.00 & 1.00 & 1.00 & 1.00 & 1.00 & 1.00 \\
& $s$ & .76 & .79 & .79 & .81 & .82 & .83 & .83 & .83 & .83 & .61 & - \\
\hline UK & $d$ & .89 & .90 & .83 & .79 & .61 & .84 & .85 & .96 & .85 & .88 & .96 \\
& $s$ & - & - & - & - & - & - & .77 & .62 & $-{ }^{b}$ & .68 & - \\
\hline Germany & $d$ & .92 & .94 & .94 & .93 & .87 & .87 & .83 & .93 & .91 & .92 & .91 \\
& $s$ & - & - & .54 & .54 & .58 & .77 & .67 & .69 & .71 & .68 & - \\
\hline India & $d$ & .77 & .81 & .70 & .68 & .72 & .64 & .60 & .58 & .57 & .59 & .58 \\
& $s$ & .26 & .21 & .18 & .29 & .20 & .36 & .53 & .53 & .63 & .73 & - \\
\hline Romania & $d$ & .88 & .88 & .84 & .83 & .80 & .79 & .82 & .83 & .79 & .82 & .77 \\
& $s$ & 51 & .41 & .60 & .85 & .61 & .70 & .75 & .73 & .80 & .80 & - \\
\hline Ghana & $d$ & .10 & .26 & .26 & .24 & .24 & .25 & .27 & .26 & .26 & .25 & .26 \\
& $s$ & - & - & - & .50 & .48 & .50 & .56 & .60 & .66 & .72 & - \\
\hline Mexico & $d$ & .27 & .27 & .63 & .46 & .45 & .48 & .60 & .55 & .60 & .56 & .74 \\
& $s$ & .75 & .51 & .71 & .75 & .72 & .74 & .81 & .79 & .81 & .75 & - \\
\hline Brazil & $d$ & .69 & .68 & .63 & .64 & .65 & .63 & .57 & .67 & .65 & .72 & .69 \\
& $s$ & .17 & .20 & .27 & .42 & .79 & .71 & .58 & .80 & .78 & .76 & - \\
\hline
\end{tabular}

\section{References}

[1] Centers for Disease Control and Prevention. TB Elimination: Treatment of Latent Tuberculosis Infection (LTBI), July 2007. Accessed March 25, 2009 from: http://cdc.gov/TB/pubs/tbfactsheets/treatmentLTBI.pdf.

[2] World Health Organization. WHO position paper on BCG vaccination. Weekly Epidemiological Record, 79(4):27-38, January 2004. Accessed on May 5, 2008 from: http://www.who.int/wer.

[3] Centers for Disease Control and Prevention. The role of BCG vaccine in the prevention and control of tuberculosis in the United States. Morbidity and Mortality Weekly Report: Recommendations and Reports, 45(RR-4), 1996.

[4] World Health Organization. Global Tuberculosis Database, 2008. Accessed March 12, 2009 from: http://www.who. int/tb/country/global_tb_database/en/.

[5] World Health Organization, Department of Immunization, Vaccines and Biologicals. WHO vaccine-preventable diseases: monitoring system. 2007 Global Summary, 2007. Accessed on March 12, 2009 from: http://whqlibdoc.who. int/hq/2007/who_ivb_2007_eng.pdf.

[6] United States Census Bureau, Population Division. International Data Base, Decemeber 15, 2008. Accessed Jan 9, 2009 from http://www.census.gov/ipc/www/idb/index.html.

[7] E. Ziv, C.L. Daley, and S.M. Blower. Potential public health impact of new tuberculosis vaccines. Emerg. Inf. Dis., 10(9):1529-1535, September 2004.

[8] Ted Cohen, Caroline Colijn, Bryson Finklea, and Megan Murray. Exogenous re-infection and the dynamics of tuberculosis epidemics: local effects in a network model for transmission. J. R. Soc. Interface, 4:523-531, 2007.

[9] P.G. Smith and A.R. Moss. Tuberculosis: Pathogenesis, Protection, and Control, chapter Epidemiology of tuberculosis. ASM Press, 1994.

[10] D. Smith, E. Wiegeshaus, and V. Balasubramanian. An analysis of some hypothesis related to the Chingelput Bacille Calmette-Guérin trial. Clinical Infectious Diseases, 31 (Suppl 3):S77-80, 2000.

[11] M.G.M. Gomes, A.O. Franco, M.C. Gomes, and G.F. Medley. The reinfection threshold promotes variability in tuberculosis epidemiology and vaccine efficay. Proc. R. Soc. Lond. B, 271:617-623, 2004.

[12] D. Menzies. What does tuberculin reactivity after Bacille Calmette-Guèrin vaccination tell us? Clinical Infectious Diseases, 31(Suppl 3):S71-4, 2000.

[13] R.I. Menzies and B. Vissandjee. Effect of Bacille Calmette-Guérin vaccination on tuberculin reactivity. Am. Rev. Respir. Dis., 145:621-5, 1992. 
[14] Centers for Disease Control and Prevention. Targeted tuberculin testing and treatment of latent tuberculosis infection. Morbidity and Mortality Weekly Report: Recommendations and Reports, 49(RR-6):1-54, 2000.

[15] F. Ma. Cecilia García-Sancho, Lourdes García-García, Ma. Eugenia Jiménez-Corona, Manuel Palacios-Martínez, Leticia D. Ferreyra-Reyes, Sergio Canizales-Quintero, Bulmaro Cano-Arellano, Alfredo Ponce de León, José SifuentesOsornio, Peter Small, and Kathryn DeRiemer. Is tuberculin skin testing useful to diagnose latent tuberculosis in BCG-vaccinated children? International Journal of Epidemiology, 35:1447-1454, 2006.

[17] S Gryzbowski, GD Barnett, and K Styblo. Contact case of active pulmonary tuberculosis. International Union against Tuberculosis, 50(1):90-106, Mar 1975.

[19] T J Joos, W C Miller, and D M Murdoch. Tuberculin reactivity in bacille Calmette-Guérin vaccinated populations: a compilation of international data. Int J Tuberc Lung Dis, 10(8):883-91, Aug 2006.

[18] D-L Ling, Y-P Liaw, C-Y Lee, H-Y Lo, H-L Yang, and P-C Chan. Contact investigation for tuberculosis in taiwan contacts aged under 20 years in 2005. Int J Tuberc Lung Dis, 15(1):50-5, Jan 2011.

[16] P. Musoke Mudido, D. Guwatudde, M.K. Nakakeeto, G.B. Bukenya, D. Nsamba, J.L. Johnson, R.D. Mugerwa, J.J. Ellner, and C.C. Whalen. The effect of bacille Calmette-Guérin vaccination at birth of tuberculin skin test reactivity in Ugandan children. Int. J. Tuberc. Lung Dis., 3(10):891-895, 1999.

[21] Ibrahim O Al-Orainey. Diagnosis of latent tuberculosis: Can we do better? Ann Thorac Med, 4(1):5-9, Jan 2009.

[20] Ajit Lalvani and Manish Pareek. A 100 year update on diagnosis of tuberculosis infection. British Medical Bulletin, 93:69-84, Jan 2010.

[22] O Oxlade, K Schwartzman, and D Menzies. Interferon-gamma release assays and tb screening in high-income countries: a cost-effectiveness analysis. Int J Tuberc Lung Dis, 11(1):16-26, Jan 2007.

[23] S. Verver, R.M. Warren, N. Beyers, M. Richardson, G.D. van der Spuy, M.W. Borgdorff, D.A. Enarson, M.A. Behr, and P.D. Helden. Rate of reinfection tuberculosis after successful treatment is higher than rate of new tuberculosis. Am. J. Respir. Crit. Care. Med., 171(12):1324-5, June 2005.

[24] Centers for Disease Control and Prevention. Reported Tuberculosis in the United States, 200\%. Atlanta, GA, September 2008.

[25] D.J. Gerberry. Trade-off between bcg vaccination and the ability to detect and treat latent tuberculosis. Journal of Theoretical Biology, 261(4):548-60, 2009.

[28] G W Comstock, V T Livesay, and S F Woolpert. The prognosis of a positive tuberculin reaction in childhood and adolescence. American Journal of Epidemiology, 99(2):131-8, Feb 1974.

[27] Thomas R. Frieden, Timothy R. Sterling, Sonal S. Munsiff, Catherine J. Watt, and Christopher Dye. Tuberculosis. Lancet, 362:887-899, 2003.

[26] Douglas Young and Christopher Dye. The development and impact of tuberculosis vaccines. Cell, 124(4):683-687, 2006.

[29] S.M. Blower, A.R. McLean, T.C. Porco, P.M. Small, P.C. Hopewell, M.A. Sanchez, and A.R. Moss. The intrinsic transmission dynamics of tuberculosis epidemics. Nature Medicine, 1(8):815-821, August 1995.

[30] T.C. Porco and S.M. Blower. Quantifying the intrinsic transmission dynamics of tuberculosis epidemics. Theoretical Population Biology, 54:117-132, 1998.

[31] Mayo Foundation for Medical Education and Research. Tuberculosis, Jan 28, 2009. Accessed May 21, 2009 from: http://www.mayoclinic.com/health/tuberculosis/DS00372/.

[33] N.E. Aronson, M. Santosham, G.W. Comstock, R.S. Howard, L.H. Moulton, E.R. Rhoades, and L.H. Harrison. Longterm efficacy of BCG vaccine in American Indians and Alaska Natives: A 60-year follow-up study. JAMA, 291(17):20862091, 2004.

[32] J.A.C. Sterne, L.C. Rodrigues, and I.N. Guedes. Does the efficacy of BCG decline with time since vaccination? Int. J. Tuberc. Lung Dis., 2(3):200-207, 1998.

[34] Christopher Dye, Geoffrey P. Garnett, Karen Sleeman, and Brian G. Williams. Prospects for worldwide tuberculosis control under the WHO DOTS strategy. The Lancet, 352:1886-1891, 1998.

[35] Christopher Dye, Suzanne Scheele, Paul Dolin, Vikram Pathania, and Mario C. Raviglione. Global burden of tuberculosis: Estimated incidence, prevalence, and mortality by country. JAMA, 282:677-686, 1999.

[36] World Health Organization. Tuberculosis factsheet No. 104, March 2007. Accessed May 10, 2009 from: http://www. who.int/mediacentre/factsheets/fs104/en/index.html.

[37] Diane E. Bennett, Jeanne M. Courval, Ida Onorato, Tracy Agerton, Judy D. Gibson, Lauren Lambert, Geraldine M. McQuillan, Brenda Lewis, Thomas R. Navin, and Kenneth G. Castro. Prevalence of tuberculosis infection in the United States population. Am. J. Respir. Crit. Care Med., 177:348-355, 2008.

[38] Timothy R. Sterling, James Bethel, Stefan Goldberg, Paul Weinfurter, Lourdes Yun, C. Robert Horsburgh, and the Tuberculosis Epidemiologic Studies Consortium. The scope and and impact of treatment of latent tuberculosis infection in the United States and Canada. Am. J. Respir. Crit. Care Med., 173:927-931, 2006.

[40] Anneke C Hesseling, Ben J Marais, Robert P Gie, H Simon Schaaf, Paul E M Fine, Peter Godfrey-Faussett, and Nulda Beyers. The risk of disseminated Bacille Calmette-Guerin (BCG) disease in HIV-infected children. Vaccine, 25(1):14-8, Jan 2007.

[39] World Health Organization. Revised BCG vaccination guidelines for children with HIV infection. pages 1-4, Apr 2007.

[41] G. Elzinga, M.C. Raviglione, and D. Maher. Scale up: meeting targets in global tuberculosis control. Lancet, 363:814$819,2004$. 